Subscriber access provided by SCD Université de Rennes1 | SCD de l'Université de Rennes 1

\title{
Note
}

\section{Synthesis of Phenanthrothiazoles and 1,2-Di(heteroaryl)benzenes Through Successive Pd-Catalyzed Direct Arylations}

\author{
Xinzhe Shi, Jean-François Soulé, and Henri Doucet
}

J. Org. Chem., Just Accepted Manuscript • DOI: 10.1021/acs.joc.6b03023 • Publication Date (Web): 08 Mar 2017

Downloaded from http://pubs.acs.org on March 9, 2017

\section{Just Accepted}

"Just Accepted" manuscripts have been peer-reviewed and accepted for publication. They are posted online prior to technical editing, formatting for publication and author proofing. The American Chemical Society provides "Just Accepted" as a free service to the research community to expedite the dissemination of scientific material as soon as possible after acceptance. "Just Accepted" manuscripts appear in full in PDF format accompanied by an HTML abstract. "Just Accepted" manuscripts have been fully peer reviewed, but should not be considered the official version of record. They are accessible to all readers and citable by the Digital Object Identifier (DOI®). "Just Accepted" is an optional service offered to authors. Therefore, the "Just Accepted" Web site may not include all articles that will be published in the journal. After a manuscript is technically edited and formatted, it will be removed from the "Just Accepted" Web site and published as an ASAP article. Note that technical editing may introduce minor changes to the manuscript text and/or graphics which could affect content, and all legal disclaimers and ethical guidelines that apply to the journal pertain. ACS cannot be held responsible for errors or consequences arising from the use of information contained in these "Just Accepted" manuscripts. 


\title{
Synthesis of Phenanthrothiazoles and 1,2-Di(heteroaryl)benzenes Through
}

\section{Successive Pd-Catalyzed Direct Arylations}

\author{
Xinzhe Shi, ${ }^{\ddagger}$ Jean-François Soulé, ${ }^{\ddagger *}$ and Henri Doucet ${ }^{\star \star *}$ \\ FInstitut des Sciences Chimiques de Rennes, UMR 6226 CNRS-Université de Rennes \\ "Organométalliques: Matériaux et Catalyse", Campus de Beaulieu, 35042 Rennes, France.
}

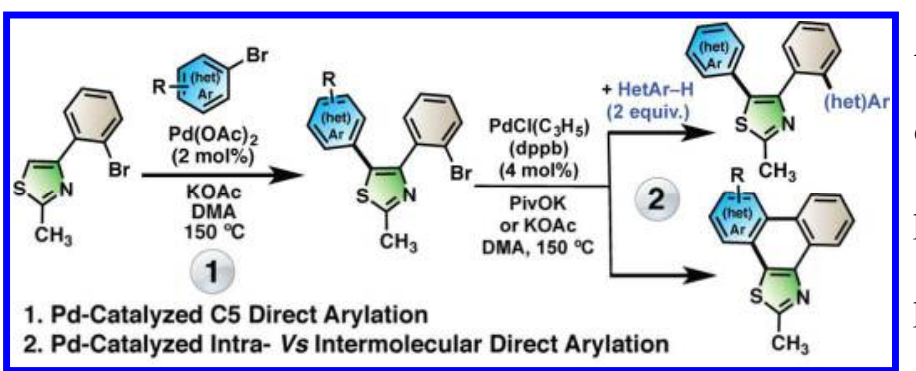

Abstract: Palladium-catalyzed direct arylation of 4-(2-bromophenyl)-2-methylthiazole proceeds in high efficiency at the thiazolyl C5 position using aryl bromides as aryl source. This transformation provides a simple access to 4-(2-bromophenyl)-2-methyl-5-arylthiazoles, which could be further converted into phenanthrothiazoles via palladium-catalyzed intramolecular direct arylation. When the direct arylation of 4-(2-bromophenyl)-2-methyl-5-arylthiazoles is conducted in the presence of an external heteroarene such as thiazoles, thiophenes, or imidazo[1,2-a]pyridines, the intermolecular arylation of such external heteroarenes proceeds faster than the intramolecular reaction, allowing the formation of 1,2-di(heteroaryl)benzene derivatives.

Polyaromatic hydrocarbons are an important class of molecules that find applications in organic electronics and optoelectronics due to their unique $\pi-\pi$ stacking features in solid states. ${ }^{1}$ The incorporation of heteroatoms such as sulfur or nitrogen, in such polyaromatic hydrocarbon structures induces a positive switch of their chemical and/or physical properties. As examples, some thiopheneanalogues of polyaromatic hydrocarbons found applications in solar cell systems, ${ }^{2}$ as motifs in helicenes, ${ }^{3}$ 
and in nanographenes. ${ }^{4}$ Some nitrogen-containing polycyclic aromatic hydrocarbons displayed unprecedented luminescence properties and represent promising candidates for fabricating organic optoelectronic devices. ${ }^{5}$ On the other hand, the thiazole moiety is present in a number of important natural products with high pharmacological activities. ${ }^{6}$ The synthesis of phenanthroid-fused thiazoles is poorly developed, albeit some phenanthrothiazoles display interesting potential pharmacological activity. ${ }^{7}$ One of the major reason of the limited information concerning phenanthrothiazole derivatives probably arises from the scarce synthetic routes described in the literature. Therefore, the discovery of straightforward synthetic routes for access to phenanthrothiazoles remains an important challenge for both academic research groups and for chemical companies.

Domínguez, Tellitu and co-worker reported the synthesis of phenanthrothiazoles through the oxidative cyclization of 4,5-diarylthiazole using a stoichiometric amount of phenyliodine(III)bis(trifluoroacetate) reagent. ${ }^{8}$ A few example of phenanthrothiazoles synthesis by pyrolysis of 1,2,4-triazine ring under hash reaction conditions (i.e. up to $350{ }^{\circ} \mathrm{C}$ ) were also reported. ${ }^{9}$ However, with these two procedures, the substrate scope remains very limited due to both challenging access to starting materials and poor functional group tolerance. During the last decades, transition-metal catalyzed direct $\mathrm{C}-\mathrm{H}$ bond functionalization has emerged as a powerful methodology for the formation of $\mathrm{C}-\mathrm{C}$ bonds, ${ }^{10}$ including direct arylations, ${ }^{11} \mathrm{C}$-heteroatom bonds, ${ }^{12}$ using palladium catalysis, ${ }^{13}$ ruthenium, ${ }^{14}$ or other metals, ${ }^{15}$ directed $\mathrm{C}-\mathrm{H}$ bonds, ${ }^{16}$ oxidative couplings,${ }^{17}$ and it was also apply to the straightforward synthesis and the easy modifications of organic molecules. ${ }^{18}$ In 2013, Nishihara and co-workers reported the synthesis of polyaromatic hydrocarbon such as triphenylenes through cross-coupling followed by annulation reaction of ortho-bromobenzylalcohols. ${ }^{19}$ A similar strategy was employed by Greaney and co-workers using 2-bromophenylboronic esters. ${ }^{20} \mathrm{C}-\mathrm{H}$ bond arylation was recently transfer to the synthesis of polyaromatic hydrocarbons containing heteroatoms. As example, phenanthro $[9,10-b]$ thiophene were synthetized by palladium-catalyzed oxidative intramolecular C-H bond arylation. ${ }^{21}$ In 2014, Bach and coworkers reported a novel synthetic route for the preparation of phenanthro[9,10-c] thiophenes involving C$\mathrm{H}$ bond activation, Suzuki cross-coupling and photocyclization. ${ }^{22}$ Recently, we have reported the 
synthesis of thiophene-analogues of polyaromatic hydrocarbons in two operations from thiophenes, 2bromobenzenesulfonyl chlorides and aryl bromides. ${ }^{23}$ The C3-desulfitative arylation of thiophenes provides a straightforward access to 4 -(2-bromophenyl)thiophene derivatives, ${ }^{24}$ which could react with activated aryl bromides in $\mathrm{Pd}$-promoted two-fold $\mathrm{C}-\mathrm{H}$ bond arylation to allow to the formation of phenanthro[9,10- $b]$ thiophenes. We employed a similar synthetic scheme for the elaboration of mediumsize heterocycles with a bridgehead nitrogen atom. ${ }^{25}$ However, to the best of our knowledge, there is no example of palladium-catalyzed successive $\mathrm{C}-\mathrm{H}$ bond arylation reactions for the formation of phenanthrothiazoles.

Our investigations started with the evaluation of the reactivity of commercially available 4-(2bromophenyl)-2-methylthiazole in the presence of 4-bromobenzonitrile under our previously optimized reaction conditions for the synthesis of $\mathrm{C} 5$-arylated thiophenes ${ }^{23,}{ }^{26}$ (i.e., $2 \mathrm{~mol} \% \mathrm{PdCl}_{(}\left(\mathrm{C}_{3} \mathrm{H}_{5}\right)(\mathrm{dppb})$ as catalyst in the presence of PivOK as base in DMA at $150{ }^{\circ} \mathrm{C}$ ). Interestingly, the intermolecular palladiumcatalyzed direct arylation proceeded to give $\mathbf{1}$ in good yield, without the cleavage of the phenyl $\mathrm{C}-\mathrm{Br}$ bond. However, under these conditions, no formation of phenanthrothiazole 14, which would result from a palladium-catalyzed one-pot two-fold direct arylation was observed. This reactivity might be explained by a slower oxidative addition rate to palladium( 0 ) of a bromobenzene bearing a thiazole substituent than a bromobenzene bearing a nitrile group. The use of $\mathrm{Pd}(\mathrm{OAc})_{2}$ as catalyst instead of $\mathrm{PdCl}\left(\mathrm{C}_{3} \mathrm{H}_{5}\right)(\mathrm{dppb})$ and KOAc as base provided $\mathbf{1}$ in a similar yield. Consequently, this phosphine-free catalytic system was selected to evaluate the reactivity of 4-(2-bromophenyl)-2-methylthiazole with other aryl bromides. All the aryl bromides that we selected displayed a similar reactivity, and only the C5-arylated thiazole derivatives were obtained. Good yields in the desired 4-(2-bromophenyl)-2-methyl-5-arylthiazoles 2-5 were obtained with bromobenzenes substituted at para-position by nitro-, ester-, formyl-, or propionylsubstituents in the presence of $2 \mathrm{~mol} \%$ of phosphine-free $\mathrm{Pd}(\mathrm{OAc})_{2}$. A complex mixture was formed when 4-bromonanisole was used as the aryl source, even using $\operatorname{PdCl}\left(\mathrm{C}_{3} \mathrm{H}_{5}\right)(\mathrm{dppb})$ catalyst. We also investigated the reactivity of 4-iodoanisole, but the desired product 6 was observed in only trace amount 
by GC/MS analysis of the crude mixture with other side-products. Among them a huge amount of 4iodoanisole homocoupling prevents the isolation of $\mathbf{6}$ in a pure form. The meta-substituted electrondeficient aryl bromides, 3-bromobenzonitrile and 1-bromo-3-(trifluoromethyl)benzene, were also found to be suitable reactants, affording 7 and 8 in $62 \%$ and $54 \%$ yields, respectively. The reaction was found to be slightly sensitive to steric hindrance as the ortho-substituted electron-deficient aryl bromides 2bromobenzonitrile and 2-bromobenzaldehyde delivered the desired 4-(2-bromophenyl)-2-methyl-5arylthiazoles 9 and $\mathbf{1 0}$ in moderate yields of $42 \%$ and 48\%, respectively. The $N$-containing heteroaryl bromides, 3-bromopyridine and 3-bromoquinoline, were also successfully employed as coupling partners to afford the C5 arylated thiazoles $\mathbf{1 1}$ and $\mathbf{1 2}$ in 66\% and 53\% yields, respectively. It is important to mention that, in all the examples mentioned above, the $\mathrm{C}-\mathrm{Br}$ bond of 4-(2-bromophenyl)-2-methylthiazole remained untouched under these reaction conditions.
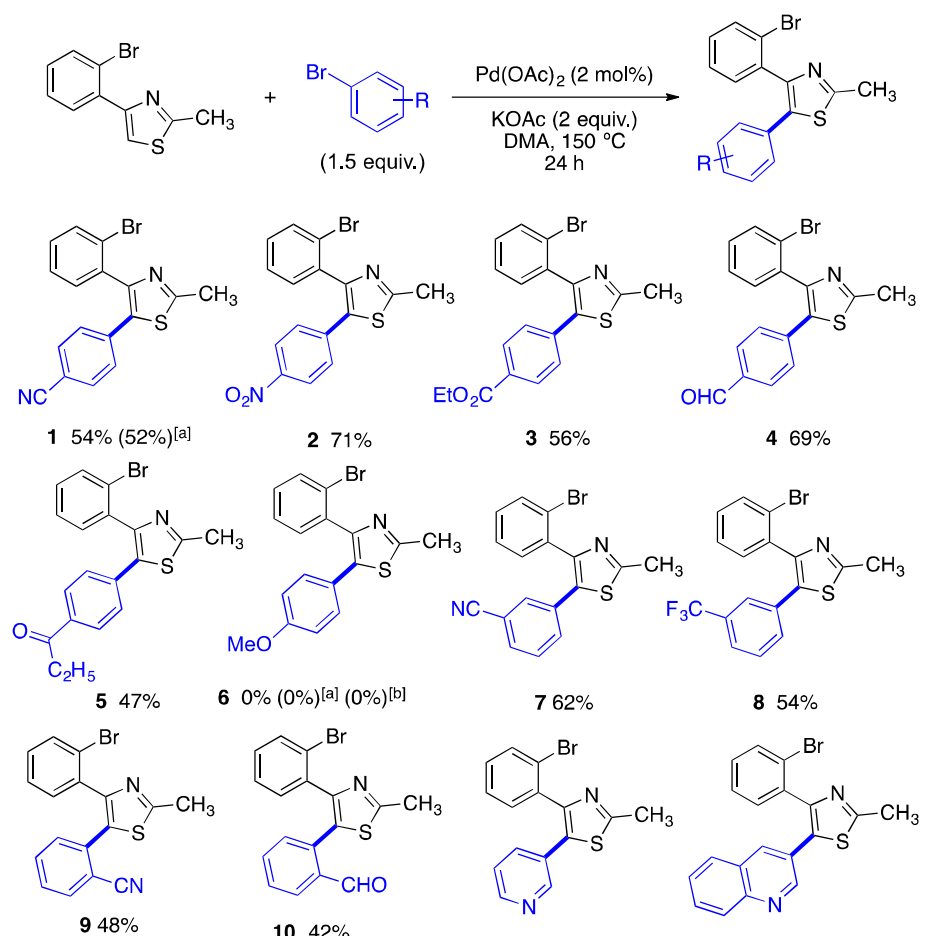

$1166 \%$

$1253 \%$

Scheme 1. Palladium-Catalyzed Direct Intermolecular C5-Arylation of 4-(2-bromophenyl)-2-methylthiazole. [a] $\mathrm{PdCl}\left(\mathrm{C}_{3} \mathrm{H}_{5}\right)(\mathrm{dppb})$ and PivOK were used instead of $\mathrm{Pd}(\mathrm{OAc})_{2}$ and KOAc. [b] The reaction is performed with 4-iodoanisole instead of 4-bromoanisole and $\mathrm{Ag}_{2} \mathrm{CO}_{3}$ as base. 
We also investigated the $\mathrm{C}-\mathrm{Br}$ bond cleavage selectivity using 4-(4-bromophenyl)-2-methylthiazole (Scheme 2). In the presence of 4-bromobenzonitrile, under the optimized reaction conditions (i.e., 2 $\mathrm{mol} \% \mathrm{Pd}(\mathrm{OAc})_{2}$, KOAc as base in DMA at $\left.150^{\circ} \mathrm{C}\right)$, we obtained the formation of the $\mathrm{C} 5$ arylated product 13 without cleavage of the 4-(4-bromophenyl)-2-methylthiazole $\mathrm{C}-\mathrm{Br}$ bond. This result suggests that the selectivity of the oxidative addition to palladium $(0)$ is due to electronic effects rather than to steric effects.

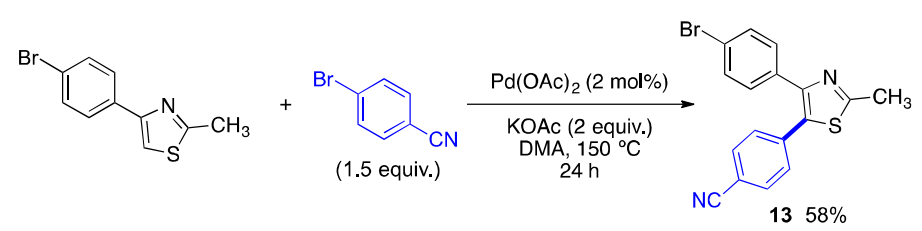

Scheme 2. Palladium-Catalyzed Direct Intermolecular C5-Arylation of 4-(4-bromophenyl)-2-methylthiazole.

In a second step, we investigated the reactivity of some of the 5-(2-bromophenyl)-2-methyl-4arylthiazoles prepared in the Scheme 1 for intramolecular direct arylation (Scheme 3). ${ }^{27}$ As expected, the compound 1 did not react in the presence of $4 \mathrm{~mol} \%$ of $\mathrm{Pd}(\mathrm{OAc})_{2}$ as catalyst associated to PivOK as base in DMA. But, we were pleased to find that $\mathbf{1}$ was cyclized into the desired phenanthrothiazole $\mathbf{1 4}$ using 4 $\operatorname{mol} \% \operatorname{PdCl}\left(\mathrm{C}_{3} \mathrm{H}_{5}\right)(\mathrm{dppb})$ as catalyst. The use of this palladium catalyst which contains a phosphine ligand, promote the oxidative addition of the 2-bromophenyl moiety. However, the use of a lower catalyst loading of $2 \mathrm{~mol} \%$ resulted in a partial conversion of $\mathbf{1}$. It is important to note that under these conditions, the reaction between 4-(2-bromophenyl)-2-methylthiazole and 4-bromobenzonitrile did not afford the product 12 resulting from a palladium-catalyzed one-pot two-fold direct arylation. Then, we evaluated, under these reaction conditions, the cyclization of few other 4-(2-bromophenyl)-2-methyl-5-arylthiazoles previously prepared. No cyclization occurred using more electron-deficient 4-(2-bromophenyl)-2-methyl5-(4-nitrophenyl)thiazole (2), and only the product $\mathbf{1 5}$ resulting from a debromination reaction was isolated in $76 \%$ yield. This result might be explained by the formation of "palladium black" due to the presence of a nitro group in the reaction mixture. Such palladium species could catalyze the debromination reaction. On the contrary, the cyclization reaction was found to be operative when the C5 aryl group on thiazole is substituted at the para position by an ester group (product 3) allowing the 
synthesis of the desired phenanthrothiazole 16 in $76 \%$ yield. The intramolecular direct arylation does not occur using 4-(4-(2-bromophenyl)-2-methylthiazol-5-yl)benzaldehyde (4), but only the degradation of 4 was observed by GC-MS and NMR analysis, including its deformylation. The cyclization of 3-(4-(2bromophenyl)-2-methylthiazol-5-yl)benzonitrile (7), containing a meta-substituent on the benzonitrile unit, lead to the single regioisomer 18 in $56 \%$ yield. The cyclization occurred at the more sterically hindered ortho-position of the cyano group. This regioselectivity is similar to our previous observations for the cyclization of 4-(2-bromophenyl)thiophene in the presence of 3-bromobenzonitrile. ${ }^{23}$ This regioselectivity might be explained by electronic factors, such as repulsing effect between sulfur atom and $\mathrm{CN}$ group ${ }^{28}$ or a directing group effect of the cyano group. In contrast, the meta-substituted 4-(2bromophenyl)-2-methyl-5-(3-(trifluoromethyl)phenyl)thiazole (8) gave a mixture of the two phenanthrothiazole regioisomers $19 \mathrm{a}$ and $\mathbf{1 9 b}$ in $44: 56$ ratio. The major product $\mathbf{1 9 b}$ results from the functionalization of the less sterically hindered $\mathrm{C}-\mathrm{H}$ bond (at para position of the $\mathrm{CF}_{3}$ ) and the minor product 19a arises from the cyclization at the ortho-position of the $\mathrm{CF}_{3}$ substituent, which is the most electron-deficient $\mathrm{C}-\mathrm{H}$ bond. A cyano substituent at ortho-position of the C5-aryl group on thiazole decreases the yield in cyclization product, as desired phenanthrothiazole $\mathbf{2 0}$ was isolated in only $45 \%$ yield. Finally, the reactivity of the compound 11, which bears a pyridine at the thiazolyl C5 position was examined in cyclization reaction. Very few examples of or intramolecular palladium-catalyzed direct arylations of substrates containing a pyridine motif have been reported. ${ }^{29}$ Using the standard reaction conditions, the cyclization reaction allowed the formation of the desired phenanthrothiazole derivative $\mathbf{2 1}$ as a single regioisomer, resulting from the activation of the $\mathrm{C} 4-\mathrm{H}$ bond of the pyridine unit, but in a very low yield. 

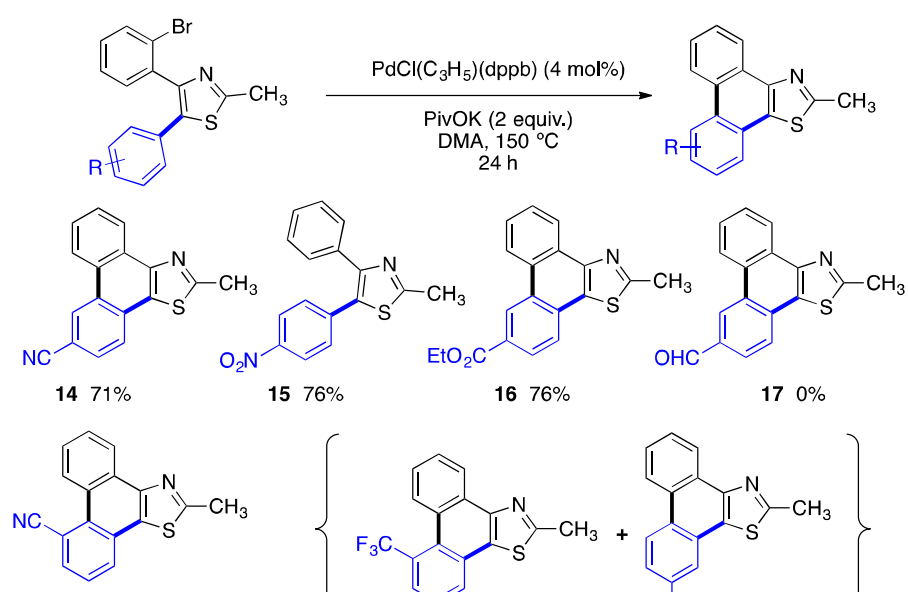

$1576 \%$

$1676 \%$

$170 \%$

$1856 \%$

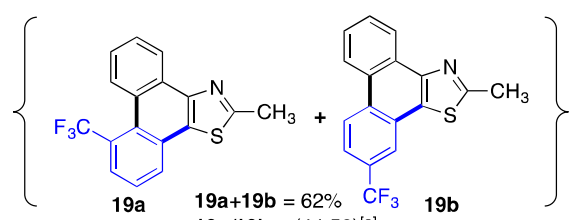

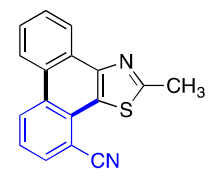

$2045 \%$ $19 a / 19 b=(44: 56)^{[a]}$

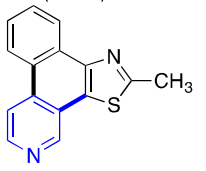

21 15\%

Scheme 3. Synthesis of Phenanthrothiazole Derivatives via Palladium-Catalyzed Direct Intramolecular Arylation of 5-(2-Bromophenyl)-2-methyl-4-arylthiazoles 1-3, 8-9 and 11. [a] ratio determined by NMR.

Next, we investigated the reactivity of some 4-(2-bromophenyl)-2-methyl-5-arylthiazoles prepared in the Scheme 1 in intermolecular direct arylation in the presence of heteroarenes (Scheme 4). Under the quite similar conditions than those employed for intramolecular direct arylation in the Scheme 2, namely, 4 $\mathrm{mol} \% \mathrm{PdCl}\left(\mathrm{C}_{3} \mathrm{H}_{5}\right)(\mathrm{dppb})$ associated to KOAc as base in DMA, we found that 4-(2-bromophenyl)-2methyl-5-arylthiazole 2 in the presence of 1.5 equivalents of 2-ethyl-4-methylthiazole lead exclusively to the polyaromatic compound $\mathbf{2 2}$ in 79\% yield, without formation of the phenanthrothiazole $\mathbf{1 4}$. This result indicates that the palladium-catalyzed intermolecular arylation proceeds faster than the intramolecular reaction. It confirms that the thiazolyl $\mathrm{C}-\mathrm{H}$ bond is more reactive than the $\mathrm{C}-\mathrm{H}$ bond of the nitrobenzene in palladium-catalyzed direct arylation. Similar results were obtained with the other 4-(2-bromophenyl)2-methyl-5-arylthiazoles $\mathbf{5}, 7$ to furnish the products $\mathbf{2 3}$ and $\mathbf{2 4}$ in good yields. Starting from 2-(4-(2bromophenyl)-2-methylthiazol-5-yl)benzaldehyde (10) and 2-ethyl-4-methylthiazole, the intermolecular direct arylation reaction proceeded in low yield without formation of intramolecular cyclizing product, but the presence of a huge amount of degradation product including one coming from a deformylation was observed. In contrast to the cyclization reaction, in which pyridine or quinoline substituents as C5 
position of the thiazole decreased drastically their reactivity, such $N$-containing heteroaryls does not affect the yields for the formation of the derivatives 26 and $\mathbf{2 7}$, which were obtained in $82 \%$ and $85 \%$ yields from the compounds $\mathbf{1 1}$ and $\mathbf{1 2}$, respectively. Using 2-isobutylthiazole, the intermolecular reaction also proceeded faster than the cyclization reaction to afford the compound $\mathbf{2 8}$ in good yield. When $n$ pentylthiophene was used as heteroarene with 12, again only the intermolecular reaction was observed to afford the C5-arylated thiophene $\mathbf{2 9}$ in 70\% yield. Finally, using imidazo[1,2-b]pyridine, which is also a very reactive substrate in palladium-catalyzed $\mathrm{C}-\mathrm{H}$ bond arylation, again only the intermolecular coupling was observed allowing the formation of $\mathbf{3 0}$ in $82 \%$ yield.

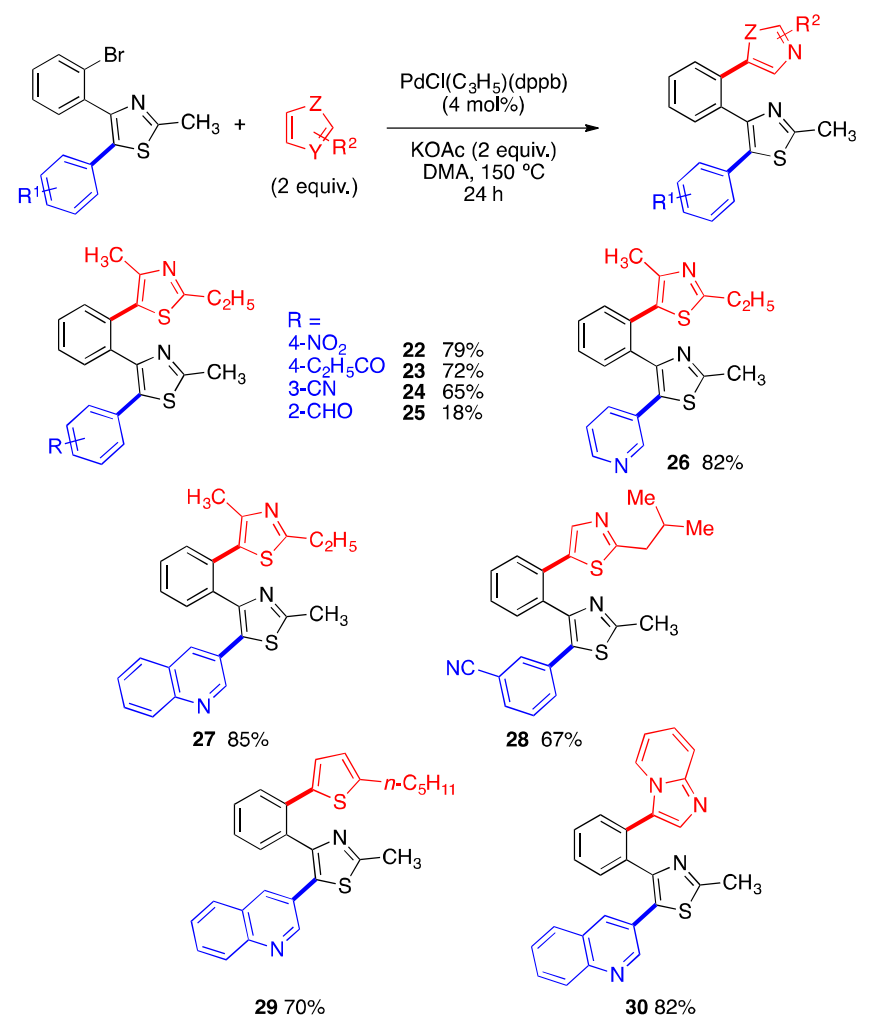

Scheme 4. Synthesis of 1,2-Dithiazolyarenes Derivatives via Palladium-Catalyzed Direct Intermolecular Arylation of 4-(2-Bromophenyl)-2-methyl-5-arylthiazoles 2, 5, 7, and 10-12.

In summary, we have discovered that in contrast to the reaction between 4-(2-bromophenyl)thiophene and electron-deficient aryl bromides, in which palladium-catalyzed one-pot cascade two-fold direct arylations to allow the synthesis of phenanthro[9,10-b]thiophenes, the reaction with 4-(2-bromophenyl)-2methylthiazole stopped after the first direct arylation. This procedure tolerates a wide variety of 
substituents on the aryl bromide such as nitro, cyano, ester, ketone, formyl, trifluoromethyl and also pyridine derivatives. The cyclization occurred in a second step using $\operatorname{PdCl}\left(\mathrm{C}_{3} \mathrm{H}_{5}\right)(\mathrm{dppb})$ as catalyst. We also demonstrated that $\operatorname{PdCl}\left(\mathrm{C}_{3} \mathrm{H}_{5}\right)(\mathrm{dppb})$ catalyst preferentially promotes the intermolecular arylation between 4-(2-bromophenyl)-2-methyl-5-arylthiazoles and heteroarenes such as thiazoles, thiophenes, and imidazo[1,2-a]pyridines. These two steps procedures from commercially aivalable strating materials represent an economically attractive, and straightforward route for the preparation of a wide range of phenanthro-fused thiazoles and polyaryl derivatives, which could inspire the preparation of structures with applications in electronic devices or potent pharmaceuticals.

\section{EXPERIMENTAL SECTION}

All reactions were carried out under argon atmosphere with standard Schlenk techniques. DMA was was not purified before use. ${ }^{1} \mathrm{H}$ NMR spectra were recorded on $400 \mathrm{MHz}$ spectrometer. Chemical shifts $(\delta)$ were reported in parts per million relative to residual chloroform $\left(7.26 \mathrm{ppm}\right.$ for ${ }^{1} \mathrm{H} ; 77.0 \mathrm{ppm}$ for $\left.{ }^{13} \mathrm{C}\right)$, constants were reported in Hertz. ${ }^{1} \mathrm{H}$ NMR assignment abbreviations were the following: singlet (s), doublet (d), triplet (t), quartet (q), doublet of doublets (dd), doublet of triplets (dt), and multiplet (m). ${ }^{13} \mathrm{C}$ NMR spectra were recorded at $100 \mathrm{MHz}$ on the same spectrometer and reported in ppm. All reagents were weighed and handled in air.

Preparation of the $\mathbf{P d C l}\left(\mathbf{C}_{3} \mathbf{H}_{5}\right)(\mathbf{d p p b})$ catalyst: ${ }^{30}$ An oven-dried $40 \mathrm{~mL}$ Schlenk tube equipped with a magnetic stirring bar under argon atmosphere, was charged with $\left[\mathrm{Pd}\left(\mathrm{C}_{3} \mathrm{H}_{5}\right) \mathrm{Cl}\right]_{2}(182 \mathrm{mg}, 0.5 \mathrm{mmol})$ and dppb (426 mg, $1 \mathrm{mmol}) .10 \mathrm{~mL}$ of anhydrous dichloromethane were added, then, the solution was stirred at room temperature for twenty minutes. The solvent was removed in vacuum. The powder was used without purification. $\left({ }^{31} \mathrm{P} 381 \mathrm{MHz}, \mathrm{CDCl}_{3}\right) \delta=19.3$ (s).

General Procedure A (Palladium-catalyzed direct intermolecular arylation): To a $25 \mathrm{~mL}$ oven dried Schlenk tube 4-(2-bromophenyl)-2-methylthiazole (254 mg, $1 \mathrm{mmol})$, aryl bromides (1.5 mmol, 1.5 equiv.), KOAc (196 mg, 2 mmol, 2 equiv.), DMA (4 mL) and $\mathrm{Pd}(\mathrm{OAc})_{2}(4.5 \mathrm{mg}, 0.02 \mathrm{mmol}, 2 \mathrm{~mol} \%)$ were successively added. The reaction mixture was evacuated by vacuum-argon cycles ( 5 times) and 
stirred at $150{ }^{\circ} \mathrm{C}$ (oil bath temperature) for 24 hours. After cooling the reaction at room temperature and concentration, the crude mixture was purified by flash chromatography to afford the desired arylated products.

General Procedure B (Palladium-catalyzed direct intramolecular arylation): To a $25 \mathrm{~mL}$ oven dried Schlenk tube 5-(2-bromophenyl)-2-methyl-4-arylthiazole (0.5 mmol), PivOK (140 mg, 1 mmol, 2 equiv.), DMA (4 mL) and $\mathrm{PdCl}\left(\mathrm{C}_{3} \mathrm{H}_{5}\right)(\mathrm{dppb})(12.2 \mathrm{mg}, 0.02 \mathrm{mmol}, 4 \mathrm{~mol} \%)$ were successively added. The reaction mixture was evacuated by vacuum-argon cycles (5 times) and stirred at $150{ }^{\circ} \mathrm{C}$ (oil bath temperature) for 24 hours. After cooling the reaction at room temperature and concentration, the crude mixture was purified by flash chromatography to afford the desired arylated products.

General Procedure C (Palladium-catalyzed direct intermolecular arylation): To a $25 \mathrm{~mL}$ oven dried Schlenk tube 5-(2-bromophenyl)-2-methyl-4-arylthiazole $(0.5 \mathrm{mmol})$, heteroarenes (1 mmol, 2 equiv.), KOAc (98 mg, $1 \mathrm{mmol}, 2$ equiv.) DMA (4 mL) and $\mathrm{PdCl}\left(\mathrm{C}_{3} \mathrm{H}_{5}\right)(\mathrm{dppb})(12.2 \mathrm{mg}, 0.02 \mathrm{mmol}, 4 \mathrm{~mol} \%)$ were successively added. The reaction mixture was evacuated by vacuum-argon cycles ( 5 times) and stirred at $150{ }^{\circ} \mathrm{C}$ (oil bath temperature) for 24 hours. After cooling the reaction at room temperature and concentration, the crude mixture was purified by flash chromatography to afford the desired arylated products.

4-(4-(2-Bromophenyl)-2-methylthiazol-5-yl)benzonitrile (1): Following the general procedure A using 4-(2-bromophenyl)-2-methylthiazole (254 mg, $1 \mathrm{mmol}$ ) and 4-bromobenzonitrile (273 $\mathrm{mg}, 1.5 \mathrm{mmol})$, the residue was purified by flash chromatography on silica gel (pentane-EtOAc, 80-20) to afford the desired compound 1 (192 mg, 54\%) as yellow oil: ${ }^{1} \mathrm{H}$ NMR (400 MHz, $\left.\mathrm{CDCl}_{3}\right) \delta(\mathrm{ppm}) 7.62(\mathrm{~d}, J=8.0 \mathrm{~Hz}, 1 \mathrm{H})$, $7.50(\mathrm{~d}, J=8.4 \mathrm{~Hz}, 2 \mathrm{H}), 7.34(\mathrm{~d}, J=4.2 \mathrm{~Hz}, 2 \mathrm{H}), 7.28-7.24(\mathrm{~m}, 3 \mathrm{H}), 2.79(\mathrm{~s}, 3 \mathrm{H}) .{ }^{13} \mathrm{C}$ NMR $(75 \mathrm{MHz}$ $\left.\mathrm{CDCl}_{3}\right) \delta(\mathrm{ppm}) 165.2,150.2,136.4,136.0,133.3,132.8,132.4,131.8,130.4,128.8,127.7,123.6,118.5$ 111.2, 19.4. Elemental analysis: calcd (\%) for $\mathrm{C}_{17} \mathrm{H}_{11} \mathrm{BrN}_{2} \mathrm{~S}$ (355.25): C 57.48, H 3.12; found: C 57.56, $\mathrm{H}$ 3.01. MS (IE) $=(\mathrm{M}+1) 356 \mathrm{~m} / \mathrm{z}$. 
4-(2-Bromophenyl)-2-methyl-5-(4-nitrophenyl)thiazole (2): Following the general procedure A using 4-(2-bromophenyl)-2-methylthiazole (254 mg, $1 \mathrm{mmol}$ ) and 1-bromo-4-nitrobenzene (303 mg, $1.5 \mathrm{mmol}$ ), the residue was purified by flash chromatography on silica gel (pentane-EtOAc, 80-20) to afford the desired compound $2(266 \mathrm{mg}, 71 \%)$ as brown oil: ${ }^{1} \mathrm{H}$ NMR $\left(400 \mathrm{MHz}, \mathrm{CDCl}_{3}\right) \delta(\mathrm{ppm}) .8 .08(\mathrm{~d}, J=8.9$ $\mathrm{Hz}, 2 \mathrm{H}), 7.63(\mathrm{~d}, J=7.9 \mathrm{~Hz}, 1 \mathrm{H}), 7.37-7.34(\mathrm{~m}, 2 \mathrm{H}), 7.33-7.25(\mathrm{~m}, 3 \mathrm{H}), 2.80(\mathrm{~s}, 3 \mathrm{H}) .{ }^{13} \mathrm{C} \mathrm{NMR}(75$ $\left.\mathrm{MHz}, \mathrm{CDCl}_{3}\right) \delta(\mathrm{ppm}) 165.5,150.7,146.8,138.4,135.9,133.4,132.4,131.9,130.5,128.9,127.8,124.0$, 123.6, 19.6. Elemental analysis: calcd (\%) for $\mathrm{C}_{16} \mathrm{H}_{11} \mathrm{BrN}_{2} \mathrm{O}_{2} \mathrm{~S}$ (375.24): C 51.21, H 2.95; found: C 51.38, H 3.21. MS (IE) $=(\mathrm{M}+1) 376 \mathrm{~m} / \mathrm{z}$.

Ethyl 4-(4-(2-bromophenyl)-2-methylthiazol-5-yl)benzoate (3): Following the general procedure A using 4-(2-bromophenyl)-2-methylthiazole (254 mg, $1 \mathrm{mmol})$ and ethyl 4-bromobenzoate (344 mg, 1.5 $\mathrm{mmol}$ ), the residue was purified by flash chromatography on silica gel (pentane-EtOAc, 80-20) to afford the desired compound $3(225 \mathrm{mg}, 56 \%)$ as colorless oil: ${ }^{1} \mathrm{H}$ NMR $\left(400 \mathrm{MHz}, \mathrm{CDCl}_{3}\right) \delta(\mathrm{ppm}) 7.89(\mathrm{~d}, J=$ $8.4 \mathrm{~Hz}, 2 \mathrm{H}), 7.61(\mathrm{~d}, J=8.1 \mathrm{~Hz}, 1 \mathrm{H}), 7.39-7.29(\mathrm{~m}, 2 \mathrm{H}), 7.27-7.18(\mathrm{~m}, 3 \mathrm{H}), 4.34(\mathrm{q}, J=7.1 \mathrm{~Hz}, 2 \mathrm{H})$, $2.78(\mathrm{~s}, 3 \mathrm{H}), 1.36(\mathrm{t}, J=7.1 \mathrm{~Hz}, 3 \mathrm{H}) .{ }^{13} \mathrm{C} \mathrm{NMR}\left(75 \mathrm{MHz}, \mathrm{CDCl}_{3}\right) \delta(\mathrm{ppm}) 166.0,164.4,149.7,136.4$, $136.2,133.8,133.2,131.9,130.0,129.8,129.5,128.2,127.6,123.8,61.0,19.4,14.3$. Elemental analysis: calcd (\%) for $\mathrm{C}_{19} \mathrm{H}_{16} \mathrm{BrNO}_{2} \mathrm{~S}$ (402.31): C 56.73, $\mathrm{H}$ 4.01; found: C 56.89, H 4.22. MS (IE) = (M+1) 403 $\mathrm{m} / \mathrm{z}$.

4-(4-(2-Bromophenyl)-2-methylthiazol-5-yl)benzaldehyde (4): Following the general procedure A using 4-(2-bromophenyl)-2-methylthiazole $(254 \mathrm{mg}, 1 \mathrm{mmol})$ and 4-bromobenzaldehyde (278 $\mathrm{mg}, 1.5$ $\mathrm{mmol}$ ), the residue was purified by flash chromatography on silica gel (pentane-EtOAc, 80-20) to afford the desired compound $4(247 \mathrm{mg}, 69 \%)$ as yellow oil: ${ }^{1} \mathrm{H}$ NMR $\left(400 \mathrm{MHz}, \mathrm{CDCl}_{3}\right) \delta(\mathrm{ppm}) 9.92(\mathrm{~s}, 1 \mathrm{H})$, $7.72(\mathrm{~d}, J=8.1 \mathrm{~Hz}, 2 \mathrm{H}), 7.61(\mathrm{~d}, J=7.9 \mathrm{~Hz}, 1 \mathrm{H}), 7.40-7.27(\mathrm{~m}, 4 \mathrm{H}), 7.27-7.20(\mathrm{~m}, 1 \mathrm{H}), 2.78(\mathrm{~s}, 3 \mathrm{H})$. ${ }^{13} \mathrm{C}$ NMR $\left(75 \mathrm{MHz}, \mathrm{CDCl}_{3}\right) \delta(\mathrm{ppm}) 191.4,165.0,150.1,137.8,136.3,135.2,133.5,133.3,131.9,130.2$, 130.0, 128.8, 127.7, 123.7, 19.5. Elemental analysis: calcd (\%) for $\mathrm{C}_{17} \mathrm{H}_{12} \mathrm{BrNOS}$ (358.25): C 57.00, $\mathrm{H}$ 3.38; found: C 56.87, H 3.59. MS (IE) $=(\mathrm{M}-1) 357 \mathrm{~m} / \mathrm{z}$. 
1-(4-(4-(2-Bromophenyl)-2-methylthiazol-5-yl)phenyl)propan-1-one (5): Following the general procedure A using 4-(2-bromophenyl)-2-methylthiazole (254 mg, $1 \mathrm{mmol}$ ) and 4-bromopropiophenone (320 mg, $1.5 \mathrm{mmol}$ ), the residue was purified by flash chromatography on silica gel (pentane-EtOAc, 85 15) to afford the desired compound 5 (181 mg, 47\%) as yellow oil: ${ }^{1} \mathrm{H}$ NMR (400 $\left.\mathrm{MHz}, \mathrm{CDCl}_{3}\right) \delta(\mathrm{ppm})$ $7.81(\mathrm{~d}, J=8.1 \mathrm{~Hz}, 2 \mathrm{H}), 7.62(\mathrm{~d}, J=7.9 \mathrm{~Hz}, 1 \mathrm{H}), 7.42-7.29(\mathrm{~m}, 2 \mathrm{H}), 7.28-7.19(\mathrm{~m}, 3 \mathrm{H}), 2.93(\mathrm{q}, J=$ $7.1 \mathrm{~Hz}, 2 \mathrm{H}), 2.78(\mathrm{~s}, 3 \mathrm{H}), 1.19(\mathrm{t}, J=7.1 \mathrm{~Hz}, 3 \mathrm{H}) .{ }^{13} \mathrm{C} \mathrm{NMR}\left(75 \mathrm{MHz}, \mathrm{CDCl}_{3}\right) \delta(\mathrm{ppm}) 200.0,164.5$, $149.7,136.5,136.2,135.7,133.7,133.2,131.9,130.1,128.4,128.3,127.6,123.8,31.7,19.4,8.2$. Elemental analysis: calcd (\%) for $\mathrm{C}_{19} \mathrm{H}_{16} \mathrm{BrNOS}$ (386.31): C 59.07, H 4.17; found: C 59.21, H 4.02. MS $(\mathrm{IE})=(\mathrm{M}+1) 387 \mathrm{~m} / \mathrm{z}$.

3-(4-(2-Bromophenyl)-2-methylthiazol-5-yl)benzonitrile (7): Following the general procedure A using 4-(2-bromophenyl)-2-methylthiazole (254 mg, $1 \mathrm{mmol}$ ) and 3-bromobenzonitrile (273 $\mathrm{mg}, 1.5 \mathrm{mmol})$, the residue was purified by flash chromatography on silica gel (pentane-EtOAc, 80-20) to afford the desired compound $7(220 \mathrm{mg}, 62 \%)$ as brown oil: ${ }^{1} \mathrm{H}$ NMR (400 MHz, $\left.\mathrm{CDCl}_{3}\right) \delta(\mathrm{ppm}) 7.60(\mathrm{~d}, J=7.7 \mathrm{~Hz}, 1 \mathrm{H})$, $7.53-7.42(\mathrm{~m}, 2 \mathrm{H}), 7.40-7.28(\mathrm{~m}, 4 \mathrm{H}), 7.25(\mathrm{~d}, J=8.1 \mathrm{~Hz}, 1 \mathrm{H}), 2.77(\mathrm{~s}, 3 \mathrm{H}) .{ }^{13} \mathrm{C}$ NMR $(75 \mathrm{MHz}$, $\left.\mathrm{CDCl}_{3}\right) \delta(\mathrm{ppm}) 164.7,149.9,135.8,133.3,133.2,132.6,132.2,131.9,131.7,131.1,130.4,129.5,127.7$, 123.6, 118.2, 112.9, 19.4. Elemental analysis: calcd (\%) for $\mathrm{C}_{17} \mathrm{H}_{11} \mathrm{BrN}_{2} \mathrm{~S}$ (355.25): C 57.48, H 3.12; found: C 57.24, H 2.95. MS (IE) $=(\mathrm{M}-1) 354 \mathrm{~m} / \mathrm{z}$.

4-(2-Bromophenyl)-2-methyl-5-(3-(trifluoromethyl)phenyl)thiazole (8): Following the general procedure A using 4-(2-bromophenyl)-2-methylthiazole (254 mg, 1 mmol) and 1-bromo-3(trifluoromethyl)benzene $(338 \mathrm{mg}, 1.5 \mathrm{mmol})$, the residue was purified by flash chromatography on silica gel (pentane-EtOAc, 90-10) to afford the desired compound 8 (215 mg, 54\%) as colorless oil: ${ }^{1} \mathrm{H}$ NMR $\left(400 \mathrm{MHz}, \mathrm{CDCl}_{3}\right) \delta(\mathrm{ppm}) 7.61(\mathrm{~d}, J=7.8 \mathrm{~Hz}, 1 \mathrm{H}), 7.49-7.44(\mathrm{~m}, 1 \mathrm{H}), 7.43(\mathrm{~s}, 1 \mathrm{H}), 7.37-7.29(\mathrm{~m}$, 4H), $7.27-7.20(\mathrm{~m}, 1 \mathrm{H}), 2.78(\mathrm{~s}, 3 \mathrm{H}) .{ }^{13} \mathrm{C} \mathrm{NMR}\left(75 \mathrm{MHz}, \mathrm{CDCl}_{3}\right) \delta(\mathrm{ppm}) 164.3,149.7,136.1,133.2$, $133.1,132.6,131.9,131.5,130.9(\mathrm{q}, J=32.1 \mathrm{~Hz}), 130.1,129.1,127.6,125.2(\mathrm{q}, J=3.9 \mathrm{~Hz}), 124.4(\mathrm{q}, J=$ 
3.7 Hz), 123.9 (q, $J=272.7 \mathrm{~Hz}), 123.8$, 19.4. Elemental analysis: calcd (\%) for $\mathrm{C}_{17} \mathrm{H}_{11} \mathrm{BrF}_{3} \mathrm{NS}(398.24)$ : C 51.27, H 2.78; found: C 51.33, H 2.89. MS (IE) $=(M+1) 399 \mathrm{~m} / \mathrm{z}$.

2-(4-(2-Bromophenyl)-2-methylthiazol-5-yl)benzonitrile (9): Following the general procedure A using 4-(2-bromophenyl)-2-methylthiazole (254 mg, $1 \mathrm{mmol}$ ) and 2-bromobenzonitrile (273 $\mathrm{mg}, 1.5 \mathrm{mmol})$, the residue was purified by flash chromatography on silica gel (pentane-EtOAc, 80-20) to afford the desired compound 9 (170 mg, 48\%) as yellow oil: ${ }^{1} \mathrm{H} \mathrm{NMR}\left(400 \mathrm{MHz}, \mathrm{CDCl}_{3}\right) \delta(\mathrm{ppm}) 7.63(\mathrm{dd}, J=1.3,7.5 \mathrm{~Hz}$, 1H), $7.50(\mathrm{dd}, J=1.0,8.0 \mathrm{~Hz}, 1 \mathrm{H}), 7.45-7.32(\mathrm{~m}, 3 \mathrm{H}), 7.32-7.21(\mathrm{~m}, 2 \mathrm{H}), 7.14(\mathrm{td}, J=1.8,7.8 \mathrm{~Hz}$, $1 \mathrm{H}), 2.79(\mathrm{~s}, 3 \mathrm{H}) .{ }^{13} \mathrm{C} \mathrm{NMR}\left(75 \mathrm{MHz}, \mathrm{CDCl}_{3}\right) \delta(\mathrm{ppm}) 165.8,151.8,135.4,135.3,133.5,133.0,132.5$, $132.4,131.7,130.0,129.8,128.6,127.3,123.6,117.8,113.2,19.4$. Elemental analysis: calcd (\%) for $\mathrm{C}_{17} \mathrm{H}_{11} \mathrm{BrN}_{2} \mathrm{~S}$ (355.25): C 57.48, H 3.12; found: C 57.21, H 3.32. MS (IE) $=(\mathrm{M}+1) 356 \mathrm{~m} / \mathrm{z}$.

2-(4-(2-Bromophenyl)-2-methylthiazol-5-yl)benzaldehyde (10): Following the general procedure A using 4-(2-bromophenyl)-2-methylthiazole $(254 \mathrm{mg}, 1 \mathrm{mmol})$ and 2-bromobenzaldehyde (278 $\mathrm{mg}, 1.5$ $\mathrm{mmol}$ ), the residue was purified by flash chromatography on silica gel (pentane-EtOAc, 80-20) to afford the desired compound $10(150 \mathrm{mg}, 42 \%)$ as yellow oil: ${ }^{1} \mathrm{H}$ NMR $\left(400 \mathrm{MHz}, \mathrm{CDCl}_{3}\right) \delta(\mathrm{ppm}) 10.04(\mathrm{~s}$, 1H), $7.84(\mathrm{~d}, J=7.5 \mathrm{~Hz}, 1 \mathrm{H}), 7.54-7.46(\mathrm{~m}, 2 \mathrm{H}), 7.44-7.33(\mathrm{~m}, 2 \mathrm{H}), 7.23-7.16(\mathrm{~m}, 2 \mathrm{H}), 7.14-7.06$ (m, 1H), $2.81(\mathrm{~s}, 3 \mathrm{H}) .{ }^{13} \mathrm{C} \mathrm{NMR}\left(75 \mathrm{MHz}, \mathrm{CDCl}_{3}\right) \delta(\mathrm{ppm}) 190.8,165.7,152.0,134.9,134.3,134.3$, $133.6,133.2,132.4,132.4,130.1,129.8,129.0,127.9,127.3,123.6,19.4$. Elemental analysis: calcd (\%) for $\mathrm{C}_{17} \mathrm{H}_{12} \mathrm{BrNOS}$ (358.25): C 57.00, H 3.38; found: C 57.11, H 3.61. MS (IE) $=(\mathrm{M}-1) 357 \mathrm{~m} / \mathrm{z}$.

4-(2-Bromophenyl)-2-methyl-5-(pyridin-3-yl)thiazole (11): Following the general procedure A using 4(2-bromophenyl)-2-methylthiazole $(254 \mathrm{mg}, 1 \mathrm{mmol})$ and 3-bromopyridine $(237 \mathrm{mg}, 1.5 \mathrm{mmol})$, the residue was purified by flash chromatography on silica gel (pentane-EtOAc, 70-30) to afford the desired compound 11 (218 mg, 66\%) as brown oil: ${ }^{1} \mathrm{H}$ NMR (400 MHz, THF- $\left.d_{8}\right) \delta(\mathrm{ppm}) 8.57-8.31(\mathrm{~m}, 2 \mathrm{H})$, $7.60(\mathrm{~d}, J=7.7 \mathrm{~Hz}, 1 \mathrm{H}), 7.46(\mathrm{~d}, J=5.2 \mathrm{~Hz}, 1 \mathrm{H}), 7.41-7.10(\mathrm{~m}, 4 \mathrm{H}), 2.71(\mathrm{~s}, 3 \mathrm{H}) .{ }^{13} \mathrm{C} \mathrm{NMR}(75 \mathrm{MHz}$, THF- $\left.d_{8}\right) \delta(\mathrm{ppm}) 164.9,151.0,149.8,149.6,137.5,136.1,133.9,133.1,131.9,130.9,129.2,128.3,124.4$, 
124.2, 19.2. Elemental analysis: calcd (\%) for $\mathrm{C}_{15} \mathrm{H}_{11} \mathrm{BrN}_{2} \mathrm{~S}$ (331.23): C 54.39, H 3.35; found: C 54.67, $\mathrm{H}$ 3.19. $\mathrm{MS}(\mathrm{IE})=(\mathrm{M}+1) 332 \mathrm{~m} / \mathrm{z}$.

4-(2-Bromophenyl)-2-methyl-5-(quinolin-3-yl)thiazole (12): Following the general procedure A using 4-(2-bromophenyl)-2-methylthiazole $(254 \mathrm{mg}, 1 \mathrm{mmol})$ and 3-bromoquinoline (312 $\mathrm{mg}, 1.5 \mathrm{mmol})$, the residue was purified by flash chromatography on silica gel (pentane-EtOAc, 70-30) to afford the desired compound 12 (202 mg, 53\%) as brown oil: ${ }^{1} \mathrm{H}$ NMR (400 MHz, $\left.\mathrm{CDCl}_{3}\right) \delta(\mathrm{ppm}) 8.69(\mathrm{~d}, J=2.1 \mathrm{~Hz}, 1 \mathrm{H})$, $8.01(\mathrm{~d}, J=8.8 \mathrm{~Hz}, 1 \mathrm{H}), 7.93(\mathrm{~d}, J=1.9 \mathrm{~Hz}, 1 \mathrm{H}), 7.65(\mathrm{~s}, 2 \mathrm{H}), 7.59(\mathrm{~d}, J=8.1 \mathrm{~Hz}, 1 \mathrm{H}), 7.48(\mathrm{t}, J=7.5$ $\mathrm{Hz}, 1 \mathrm{H}), 7.38(\mathrm{dd}, J=1.6,7.6 \mathrm{~Hz}, 1 \mathrm{H}), 7.30(\mathrm{td}, J=1.0,7.1 \mathrm{~Hz}, 1 \mathrm{H}), 7.21(\mathrm{td}, J=1.7,7.8 \mathrm{~Hz}, 1 \mathrm{H}), 2.79$ (s, 3H). ${ }^{13} \mathrm{C}$ NMR $\left(75 \mathrm{MHz}, \mathrm{CDCl}_{3}\right) \delta(\mathrm{ppm})$ 164.7, 150.1, 149.8, 147.0, 136.1, 134.7, 133.3, 132.0, $131.3,130.3,129.9,129.2,127.9,127.7,127.5,127.2,125.3,123.8,19.5$. Elemental analysis: calcd (\%) for $\mathrm{C}_{19} \mathrm{H}_{13} \mathrm{BrN}_{2} \mathrm{~S}$ (381.29): C 59.85, H 3.44; found: C 60.13, H 3.58. MS (IE) $=(\mathrm{M}+1) 382 \mathrm{~m} / \mathrm{z}$.

4-(4-(4-Bromophenyl)-2-methylthiazol-5-yl)benzonitrile (13): Following the general procedure A using 4-(4-bromophenyl)-2-methylthiazole (254 mg, $1 \mathrm{mmol})$ and 4-bromobenzonitrile (273 $\mathrm{mg}, 1.5 \mathrm{mmol})$, the residue was purified by flash chromatography on silica gel (pentane-EtOAc, 80-20) to afford the desired compound 13 (206 mg, 58\%) as yellow oil: ${ }^{1} \mathrm{H}$ NMR (400 MHz, $\left.\mathrm{CDCl}_{3}\right) \delta(\mathrm{ppm}) 7.58(\mathrm{~d}, J=8.3 \mathrm{~Hz}, 2 \mathrm{H})$, $7.42(\mathrm{~d}, J=8.5 \mathrm{~Hz}, 2 \mathrm{H}), 7.38(\mathrm{~d}, J=8.3 \mathrm{~Hz}, 2 \mathrm{H}), 7.31(\mathrm{~d}, J=8.5 \mathrm{~Hz}, 2 \mathrm{H}), 2.75(\mathrm{~s}, 3 \mathrm{H}) .{ }^{13} \mathrm{C} \mathrm{NMR}(100$ $\left.\mathrm{MHz}, \mathrm{CDCl}_{3}\right) \delta(\mathrm{ppm}) 165.6,149.8,136.8,133.2,132.6,131.8,130.7,130.6,130.0,122.6,118.4,111.7$, 19.3. Elemental analysis: calcd (\%) for $\mathrm{C}_{17} \mathrm{H}_{11} \mathrm{BrN}_{2} \mathrm{~S}$ (355.25): C 57.48, H 3.12; found: C 57.21, H 2.89. $\operatorname{MS}(\mathrm{IE})=(\mathrm{M}+1) 356 \mathrm{~m} / \mathrm{z}$.

2-Methylphenanthro[9,10-d]thiazole-9-carbonitrile (14): Following the general procedure B using 4(4-(2-bromophenyl)-2-methylthiazol-5-yl)benzonitrile (1) (178 $\mathrm{mg}, 0.5 \mathrm{mmol})$, the residue was purified by flash chromatography on silica gel (pentane- $\left.\mathrm{Et}_{2} \mathrm{O}, 85-15\right)$ to afford the desired compound 14 (97 $\mathrm{mg}$, $71 \%)$ as a white solid $\left(\mathrm{mp}=251-254{ }^{\circ} \mathrm{C}\right):{ }^{1} \mathrm{H}$ NMR $\left(400 \mathrm{MHz}, \mathrm{CDCl}_{3}\right) \delta(\mathrm{ppm}) 8.99(\mathrm{~s}, 1 \mathrm{H}), 8.82(\mathrm{~d}, J=$ $8.9 \mathrm{~Hz}, 1 \mathrm{H}), 8.62(\mathrm{~d}, J=7.7 \mathrm{~Hz}, 1 \mathrm{H}), 7.97(\mathrm{~d}, J=8.3 \mathrm{~Hz}, 1 \mathrm{H}), 7.83-7.73(\mathrm{~m}, 3 \mathrm{H}), 2.99(\mathrm{~s}, 3 \mathrm{H}) .{ }^{13} \mathrm{C}$ $\operatorname{NMR}\left(75 \mathrm{MHz}, \mathrm{CDCl}_{3}\right) \delta(\mathrm{ppm}) 167.7,150.7,129.8,129.7,129.0,128.8,128.7,128.6,128.5,128.3$, 
127.6, 126.7, 125.0, 123.0, 119.3, 109.5, 20.3. Elemental analysis: calcd (\%) for $\mathrm{C}_{17} \mathrm{H}_{10} \mathrm{~N}_{2} \mathrm{~S}(274.34)$ : C 74.43, H 3.67; found: C 74.59, H 3.72. MS (IE) $=(\mathrm{M}) 274 \mathrm{~m} / \mathrm{z}$.

2-Methyl-5-(4-nitrophenyl)-4-phenylthiazole (15) Following the general procedure B using 4-(2bromophenyl)-2-methyl-5-(4-nitrophenyl)thiazole (2) (188 $\mathrm{mg}, 0.5 \mathrm{mmol})$, the residue was purified by flash chromatography on silica gel (pentane- $\left.\mathrm{Et}_{2} \mathrm{O}, 80-20\right)$ to afford the desired compound 15 (112 mg, $76 \%)$ as a yellow solid $\left(\mathrm{mp}=133-136{ }^{\circ} \mathrm{C}\right):{ }^{1} \mathrm{H}$ NMR $\left(400 \mathrm{MHz}, \mathrm{CDCl}_{3}\right) \delta(\mathrm{ppm}) 8.14(\mathrm{~d}, J=8.9 \mathrm{~Hz}, 2 \mathrm{H})$, 7.48-7.46 (m, 2H), $7.46-7.43(\mathrm{~m}, 2 \mathrm{H}), 7.36-7.30(\mathrm{~m}, 3 \mathrm{H}), 2.79(\mathrm{~s}, 3 \mathrm{H}) .{ }^{13} \mathrm{C} \mathrm{NMR}\left(75 \mathrm{MHz}, \mathrm{CDCl}_{3}\right) \delta$ (ppm) 165.6, 151.7, 147.0, 139.1, 134.2, 130.0, 129.8, 129.1, 128.7, 128.5, 124.0, 19.4. Elemental analysis: calcd (\%) for $\mathrm{C}_{16} \mathrm{H}_{12} \mathrm{~N}_{2} \mathrm{O}_{2} \mathrm{~S}$ (296.34): C 64.85, H 4.08; found: C 65.11, H 4.23. MS (IE) = (M) $296 \mathrm{~m} / \mathrm{z}$.

Ethyl 2-methylphenanthro[9,10-d] thiazole-9-carboxylate (16): Following the general procedure B using 4 ethyl 4-(4-(2-bromophenyl)-2-methylthiazol-5-yl)benzoate (3) (201 mg, $0.5 \mathrm{mmol})$, the residue was purified by flash chromatography on silica gel (pentane- $\mathrm{CH}_{2} \mathrm{Cl}_{2}, 40-60$ ) to afford the desired compound $16(122 \mathrm{mg}, 76 \%)$ as a yellow solid $\left(\mathrm{mp}=175-178{ }^{\circ} \mathrm{C}\right)::{ }^{1} \mathrm{H} \mathrm{NMR}\left(400 \mathrm{MHz}, \mathrm{CDCl}_{3}\right) \delta(\mathrm{ppm})$ $9.40(\mathrm{~s}, 1 \mathrm{H}), 8.86-8.66(\mathrm{~m}, 2 \mathrm{H}), 8.21(\mathrm{dd}, J=1.4,8.3 \mathrm{~Hz}, 1 \mathrm{H}), 7.91(\mathrm{~d}, J=8.3 \mathrm{~Hz}, 1 \mathrm{H}), 7.81-7.63(\mathrm{~m}$, $2 \mathrm{H}), 4.50(\mathrm{q}, J=7.1 \mathrm{~Hz}, 2 \mathrm{H}), 2.97(\mathrm{~s}, 3 \mathrm{H}), 1.50(\mathrm{t}, J=7.1 \mathrm{~Hz}, 3 \mathrm{H}) .{ }^{13} \mathrm{C} \mathrm{NMR}\left(75 \mathrm{MHz}, \mathrm{CDCl}_{3}\right) \delta(\mathrm{ppm})$ $166.9,166.6,150.1,130.2,130.1,129.8,128.2,128.1,127.9,127.8,127.4,127.2,125.9,125.8,124.8$, 123.3, 61.3, 20.2, 14.5. Elemental analysis: calcd (\%) for $\mathrm{C}_{19} \mathrm{H}_{15} \mathrm{NO}_{2} \mathrm{~S}$ (321.39): C 71.01, H 4.70; found: C 70.87, H 4.65. MS (IE) $=(\mathrm{M}+1) 322 \mathrm{~m} / \mathrm{z}$.

2-Methylphenanthro[9,10-d]thiazole-8-carbonitrile (18): Following the general procedure B using 3(4-(2-bromophenyl)-2-methylthiazol-5-yl)benzonitrile (7) (178 $\mathrm{mg}, 0.5 \mathrm{mmol})$, the residue was purified by flash chromatography on silica gel (pentane- $\mathrm{CH}_{2} \mathrm{Cl}_{2}, 20-80$ ) to afford the desired compound 18 (77 $\mathrm{mg}$, $56 \%)$ as a white solid $\left(\mathrm{mp}=231-234{ }^{\circ} \mathrm{C}\right)::{ }^{1} \mathrm{H} \mathrm{NMR}\left(400 \mathrm{MHz}, \mathrm{CDCl}_{3}\right) \delta(\mathrm{ppm}) 9.77(\mathrm{~d}, J=8.8 \mathrm{~Hz}, 1 \mathrm{H})$, $8.85(\mathrm{dd}, J=1.5,7.9 \mathrm{~Hz}, 1 \mathrm{H}), 8.11(\mathrm{dd}, J=1.3,8.0 \mathrm{~Hz}, 1 \mathrm{H}), 8.03(\mathrm{dd}, J=1.3,7.4 \mathrm{~Hz}, 1 \mathrm{H}), 7.86-7.71$ (m, 2H), $7.63(\mathrm{t}, J=7.7 \mathrm{~Hz}, 1 \mathrm{H}), 2.96(\mathrm{~s}, 3 \mathrm{H}) .{ }^{13} \mathrm{C} \mathrm{NMR}\left(75 \mathrm{MHz}, \mathrm{CDCl}_{3}\right) \delta(\mathrm{ppm}) 166.7,149.5,135.5$, 
$131.0,130.3,128.9,128.9,128.4,128.4,128.0,127.2,126.3,125.2,124.9,121.3,108.4,20.2$. Elemental analysis: calcd (\%) for $\mathrm{C}_{17} \mathrm{H}_{10} \mathrm{~N}_{2} \mathrm{~S}$ (274.34): C 74.43, H 3.67; found: C 74.63, H 3.55. MS (IE) = (M) 274 $\mathrm{m} / \mathrm{z}$.

2-Methyl-8-(trifluoromethyl)phenanthro[9,10-d] thiazole

(19a) and 2-Methyl-10(trifluoromethyl)phenanthro[9,10-d]thiazole (19b): Following the general procedure B using 3-(4-(2bromophenyl)-2-methylthiazol-5-yl)benzonitrile (8) (199 $\mathrm{mg}, 0.5 \mathrm{mmol})$, the residue was purified by flash chromatography on silica gel (pentane- $\left.\mathrm{CH}_{2} \mathrm{Cl}_{2}, 60-40\right)$ to afford the desired compound $19(98 \mathrm{mg}, 62 \%)$ as a mixture of the two regioisomers in $44: 56$ ratio as a white solid $\left(\mathrm{mp}=111-115^{\circ} \mathrm{C}\right)::{ }^{1} \mathrm{H} \mathrm{NMR}(400 \mathrm{MHz}$, $\left.\mathrm{CDCl}_{3}\right) \delta(\mathrm{ppm}) 8.80(\mathrm{dd}, J=1.3,8.0 \mathrm{~Hz}, 1 \mathrm{H}, \mathbf{1 9 b}), 8.78-8.71(\mathrm{~m}, 2 \mathrm{H}, \mathbf{1 9 a}+\mathbf{1 9 b}), 8.67(\mathrm{~d}, J=8.7 \mathrm{~Hz}, 1 \mathrm{H}, \mathbf{1 9 b})$, 8.57 (d, $J=8.0 \mathrm{~Hz}, 1 \mathrm{H}, \mathbf{1 9 b}), 8.06$ (s, 1H, 19b), 8.04 (d, $J=8.2 \mathrm{~Hz}, 1 \mathrm{H}, 19 \mathrm{a}), 7.99$ (d, $J=7.9 \mathrm{~Hz}, 1 \mathrm{H}, 19 \mathbf{a}), 7.78-$ $7.66(\mathrm{~m}, 5 \mathrm{H}, \mathbf{1 9 a}+\mathbf{1 9 b}), 7.64-7.57(\mathrm{~m}, 1 \mathrm{H}, \mathbf{1 9 a}), 2.93$ (s, 3H, 19b), 2.92 (s, 3H, 19a). ${ }^{13} \mathrm{C} \mathrm{NMR}(75 \mathrm{MHz}$, $\left.\mathrm{CDCl}_{3}\right) \delta(\mathrm{ppm}) .166 .3,166.2,149.4,149.1,130.7(\mathrm{q}, J=2.1 \mathrm{~Hz}), 130.5(\mathrm{q}, J=30,1 \mathrm{~Hz}), 130.0,129.5,129.1$, $128.9,128.9(\mathrm{q}, J=32.1 \mathrm{~Hz}), 128.8,128.7,128.5,128.4,128.1,127.9,127.3,127.1(\mathrm{q}, J=8.2 \mathrm{~Hz}), 127.1,126.6$, 126.0, 125.9, 125.4 (q, $J=271.5 \mathrm{~Hz}), 124.8,124.4,124.2,124.1$ (q, $J=271.5 \mathrm{~Hz}), 123.3,122.8(\mathrm{q}, J=4.0 \mathrm{~Hz})$, 122.1 (q, $J=4.0 \mathrm{~Hz}$ ), 20.1, 20.1. Elemental analysis: calcd (\%) for $\mathrm{C}_{17} \mathrm{H}_{10} \mathrm{~F}_{3} \mathrm{NS}$ (317.33): C 64.35, H 3.18; found: C 64.49, H 3.11. MS (IE) = (M) 317 m/z.

2-Methylphenanthro[9,10-d]thiazole-11-carbonitrile (20): Following the general procedure B using 2(4-(2-bromophenyl)-2-methylthiazol-5-yl)benzonitrile (9) (178 $\mathrm{mg}, 0.5 \mathrm{mmol})$, the residue was purified by flash chromatography on silica gel (pentane- $\mathrm{CH}_{2} \mathrm{Cl}_{2}, 10-90$ ) to afford the desired compound 20 (61 mg, $45 \%)$ as a yellow solid $\left(\mathrm{mp}=150-153{ }^{\circ} \mathrm{C}\right)::{ }^{1} \mathrm{H}$ NMR $\left(400 \mathrm{MHz}, \mathrm{DMSO}-d_{6}\right) \delta(\mathrm{ppm}) 9.24(\mathrm{~d}, J=8.3 \mathrm{~Hz}$, 1H), $8.89(\mathrm{~d}, J=7.5 \mathrm{~Hz}, 1 \mathrm{H}), 8.71(\mathrm{~d}, J=7.5 \mathrm{~Hz}, 1 \mathrm{H}), 8.25(\mathrm{~d}, J=7.4 \mathrm{~Hz}, 1 \mathrm{H}), 7.91-7.73(\mathrm{~m}, 3 \mathrm{H}), 2.96$ $(\mathrm{s}, 3 \mathrm{H}) .{ }^{13} \mathrm{C}$ NMR (100 MHz, DMSO-d $d_{6} \delta(\mathrm{ppm}) 168.8,151.1,135.4,130.1,129.3,129.2,129.1,128.4$, $127.8,127.5,127.4,126.7,124.9,124.3,119.9,107.4,19.9$. Elemental analysis: calcd (\%) for $\mathrm{C}_{17} \mathrm{H}_{10} \mathrm{~N}_{2} \mathrm{~S}$ (274.34): C 74.43, H 3.67; found: C 74.59, H 3.72. MS (IE) $=(\mathrm{M}) 274 \mathrm{~m} / \mathrm{z}$. 
2-Methylbenzo $[\boldsymbol{f}]$ thiazolo[4,5-h]isoquinoline (21): Following the general procedure B using 4-(2Bromophenyl)-2-methyl-5-(pyridin-3-yl)thiazole (11) (166 mg, $0.5 \mathrm{mmol}, 1$ equiv.) the residue was purified by flash chromatography on silica gel (pentane-Et ${ }_{2} \mathrm{O}, 50-50$ ) to afford the desired compound 21 $(19 \mathrm{mg}, 15 \%)$ as a brown solid $\left(\mathrm{mp}=45-50{ }^{\circ} \mathrm{C}\right)::{ }^{1} \mathrm{H}$ NMR $\left(400 \mathrm{MHz}, \mathrm{CDCl}_{3}\right) \delta(\mathrm{ppm}) 9.34(\mathrm{~d}, J=8.0$ $\mathrm{Hz}, 1 \mathrm{H}), 9.02(\mathrm{~d}, J=3.3 \mathrm{~Hz}, 1 \mathrm{H}), 8.80(\mathrm{~d}, J=7.9 \mathrm{~Hz}, 1 \mathrm{H}), 8.27(\mathrm{~d}, J=7.7 \mathrm{~Hz}, 1 \mathrm{H}), 7.89-7.75(\mathrm{~m}, 2 \mathrm{H})$, $7.58(\mathrm{dd}, J=4.4,8.1 \mathrm{~Hz}, 1 \mathrm{H}), 3.01(\mathrm{~s}, 3 \mathrm{H}) .{ }^{13} \mathrm{C} \mathrm{NMR}\left(75 \mathrm{MHz}, \mathrm{CDCl}_{3}\right) \delta(\mathrm{ppm}) 166.3,148.3,145.2$, 133.0, 130.9, 130.8, 129.3, 129.0, 127.1, 125.1, 124.0, 122.2, 122.1, 118.6, 20.2. Elemental analysis: calcd (\%) for $\mathrm{C}_{15} \mathrm{H}_{10} \mathrm{~N}_{2} \mathrm{~S}$ (250.31): C 71.97, H 4.03; found: C 72.08, H 4.34. MS (IE) = (M) $250 \mathrm{~m} / \mathrm{z}$.

2-Ethyl-4-methyl-5-(2-(2-methyl-5-(4-nitrophenyl)thiazol-4-yl)phenyl)thiazole (22): Following the general procedure $\mathbf{C}$ using 4-(2-bromophenyl)-2-methyl-5-(4-nitrophenyl)thiazole (2) (188 mg, $0.5 \mathrm{mmol}$, 1 equiv.) and 2-ethyl-4-methylthiazole (127 mg, $1 \mathrm{mmol}, 2$ equiv.), the residue was purified by flash chromatography on silica gel (pentane-EtOAc, 50-50) to afford the desired compound 22 (172 $\mathrm{mg}, 79 \%)$ as a brown solid $\left(\mathrm{mp}=99-102{ }^{\circ} \mathrm{C}\right):{ }^{1} \mathrm{H}$ NMR $\left(400 \mathrm{MHz}, \mathrm{CDCl}_{3}\right) \delta(\mathrm{ppm}) 7.98(\mathrm{~d}, J=8.4 \mathrm{~Hz}, 2 \mathrm{H}), 7.71(\mathrm{~d}$, $J=7.5 \mathrm{~Hz}, 1 \mathrm{H}), 7.50$ (t, $J=7.2 \mathrm{~Hz}, 1 \mathrm{H}), 7.44(\mathrm{~d}, J=7.4 \mathrm{~Hz}, 1 \mathrm{H}), 7.26-7.24(\mathrm{~m}, 1 \mathrm{H}), 7.00(\mathrm{~d}, J=8.6 \mathrm{~Hz}$, 2H), $2.87-2.69(\mathrm{~m}, 2 \mathrm{H}), 2.77(\mathrm{~s}, 3 \mathrm{H}), 1.74(\mathrm{~s}, 3 \mathrm{H}), 1.24(\mathrm{t}, J=7.5 \mathrm{~Hz}, 3 \mathrm{H}) .{ }^{13} \mathrm{C} \mathrm{NMR}\left(75 \mathrm{MHz}, \mathrm{CDCl}_{3}\right)$ $\delta(\mathrm{ppm}) 165.9,150.5,147.9,146.9,138.6,134.7,132.0,131.9,131.3,131.2,129.2,129.2,129.1,129.0$, 128.9, 123.9, 26.8, 19.5, 15.2, 14.6. Elemental analysis: calcd (\%) for $\mathrm{C}_{22} \mathrm{H}_{19} \mathrm{~N}_{3} \mathrm{O}_{2} \mathrm{~S}_{2}$ (421.53): C 62.69, $\mathrm{H}$ 4.54; found: C 62.79, H 4.31. MS (IE) = (M) $421 \mathrm{~m} / \mathrm{z}$.

\section{1-(4-(4-(2-(2-Ethyl-4-methylthiazol-5-yl)phenyl)-2-methylthiazol-5-yl)phenyl)propan-1-one}

(23):

Following the general procedure $\mathbf{C}$ using 1-(4-(4-(2-bromophenyl)-2-methylthiazol-5-yl)phenyl)propan-1one (5) (193 mg, $0.5 \mathrm{mmol}, 1$ equiv.) and 2-ethyl-4-methylthiazole (127 mg, $1 \mathrm{mmol}, 2$ equiv.), the residue was purified by flash chromatography on silica gel (pentane-EtOAc, 50-50) to afford the desired compound $23(156 \mathrm{mg}, 72 \%)$ as a brown solid $\left(\mathrm{mp}=64-49{ }^{\circ} \mathrm{C}\right):{ }^{1} \mathrm{H} \mathrm{NMR}\left(400 \mathrm{MHz}, \mathrm{CDCl}_{3}\right) \delta(\mathrm{ppm}) 7.78$ $-7.68(\mathrm{~m}, 3 \mathrm{H}), 7.50(\mathrm{t}, J=7.0 \mathrm{~Hz}, 1 \mathrm{H}), 7.43(\mathrm{td}, J=1.3,7.5 \mathrm{~Hz}, 1 \mathrm{H}), 7.26(\mathrm{~d}, J=7.6 \mathrm{~Hz}, 1 \mathrm{H}), 6.96(\mathrm{~d}, J$ $=8.3 \mathrm{~Hz}, 2 \mathrm{H}), 2.95(\mathrm{q}, J=7.2 \mathrm{~Hz}, 2 \mathrm{H}), 2.89-2.81(\mathrm{~m}, 2 \mathrm{H}), 2.78(\mathrm{~s}, 3 \mathrm{H}), 1.75(\mathrm{~s}, 3 \mathrm{H}), 1.27(\mathrm{~d}, J=7.3$ 
$\mathrm{Hz}, 3 \mathrm{H}), 1.22(\mathrm{t}, J=7.2 \mathrm{~Hz}, 3 \mathrm{H}) .{ }^{13} \mathrm{C} \mathrm{NMR}\left(75 \mathrm{MHz}, \mathrm{CDCl}_{3}\right) \delta(\mathrm{ppm}) 200.0,164.8,149.4,147.7,136.5$, $136.5,136.3,135.6,135.2,134.4,133.3,131.7,131.2,129.2,128.6,128.3,128.2,31.8,26.6,19.3,15.1$, 14.5, 8.2. Elemental analysis: calcd (\%) for $\mathrm{C}_{25} \mathrm{H}_{24} \mathrm{~N}_{2} \mathrm{OS}_{2}$ (432.60): C 69.41, H 5.59; found: C 69.49, $\mathrm{H}$ 5.21. MS (IE) $=(\mathrm{M}) 432 \mathrm{~m} / \mathrm{z}$.

3-(4-(2-(2-Ethyl-4-methylthiazol-5-yl)phenyl)-2-methylthiazol-5-yl)benzonitrile (24): Following the general procedure $\mathbf{C}$ using 3-(4-(2-bromophenyl)-2-methylthiazol-5-yl)benzonitrile (7) (178 mg, 0.5 mmol, 1 equiv.) and 2-ethyl-4-methylthiazole (127 mg, $1 \mathrm{mmol}, 2$ equiv.), the residue was purified by flash chromatography on silica gel (pentane-EtOAc, 50-50) to afford the desired compound 24 (130 mg, $65 \%)$ as a brown solid $\left(\mathrm{mp}=47-50{ }^{\circ} \mathrm{C}\right):{ }^{1} \mathrm{H}$ NMR $\left(400 \mathrm{MHz}, \mathrm{CDCl}_{3}\right) \delta(\mathrm{ppm}) 7.70(\mathrm{dd}, J=1.4,7.6 \mathrm{~Hz}$, 1H), $7.46(\mathrm{~d}, J=7.2,16.6 \mathrm{~Hz}, 3 \mathrm{H}), 7.30-7.19(\mathrm{~m}, 2 \mathrm{H}), 7.10(\mathrm{~s}, 1 \mathrm{H}), 7.05(\mathrm{~d}, J=7.9 \mathrm{~Hz}, 1 \mathrm{H}), 2.84(\mathrm{q}, J=$ $7.5 \mathrm{~Hz}, 2 \mathrm{H}), 2.76(\mathrm{~s}, 3 \mathrm{H}), 1.74(\mathrm{~s}, 3 \mathrm{H}), 1.28(\mathrm{t}, J=7.6 \mathrm{~Hz}, 3 \mathrm{H}) .{ }^{13} \mathrm{C} \mathrm{NMR}\left(75 \mathrm{MHz}, \mathrm{CDCl}_{3}\right) \delta(\mathrm{ppm})$ $165.2,149.7,147.9,134.5,133.4,132.5,131.9,131.7,131.6,131.4,131.1,131.0,129.5,129.2,129.0$, 128.9, 118.2, 112.9, 26.8, 19.5, 15.3, 14.5. Elemental analysis: calcd (\%) for $\mathrm{C}_{23} \mathrm{H}_{19} \mathrm{~N}_{3} \mathrm{~S}_{2}$ (401.55): C 68.80, H 4.77; found: C 68.79, H 5.03. MS (IE) = (M) $401 \mathrm{~m} / \mathrm{z}$.

2-(4-(2-(2-Ethyl-4-methylthiazol-5-yl)phenyl)-2-methylthiazol-5-yl)benzaldehyde (25): Following the general procedure $\mathbf{C}$ using 2-(4-(2-bromophenyl)-2-methylthiazol-5-yl)benzaldehyde (10) (179 mg, 0.5 mmol, 1 equiv.) and 2-ethyl-4-methylthiazole (127 mg, $1 \mathrm{mmol}, 2$ equiv.), the residue was purified by flash chromatography on silica gel (pentane-EtOAc, 50-50) to afford the desired compound 25 (37 mg, 18\%) as yellow oil: ${ }^{1} \mathrm{H} \mathrm{NMR}\left(400 \mathrm{MHz}, \mathrm{CDCl}_{3}\right) \delta(\mathrm{ppm}){ }^{1} \mathrm{H} \mathrm{NMR}\left(400 \mathrm{MHz}, \mathrm{CDCl}_{3}\right) \delta 9.64(\mathrm{~s}, 1 \mathrm{H}), 7.75$ $(\mathrm{dd}, J=3.4,5.9 \mathrm{~Hz}, 1 \mathrm{H}), 7.72(\mathrm{~d}, J=7.8 \mathrm{~Hz}, 1 \mathrm{H}), 7.46(\mathrm{t}, J=7.0 \mathrm{~Hz}, 1 \mathrm{H}), 7.39-7.31(\mathrm{~m}, 3 \mathrm{H}), 7.13(\mathrm{~d}, J$ $=7.7 \mathrm{~Hz}, 1 \mathrm{H}), 6.78(\mathrm{dd}, J=3.2,5.7 \mathrm{~Hz}, 1 \mathrm{H}), 2.86(\mathrm{q}, J=7.5 \mathrm{~Hz}, 2 \mathrm{H}), 2.82(\mathrm{~s}, 3 \mathrm{H}), 1.67(\mathrm{~s}, 3 \mathrm{H}), 1.30(\mathrm{t}, J$ $=7.6 \mathrm{~Hz}, 3 \mathrm{H}) .{ }^{13} \mathrm{C} \mathrm{NMR}\left(75 \mathrm{MHz}, \mathrm{CDCl}_{3}\right) \delta(\mathrm{ppm}) 190.1,166.2,151.6,135.8,134.3,133.8,133.4$, $133.3,132.2,131.8,131.5,131.2,129.4,129.1,128.8,128.7,128.6,127.8,125.5,26.6,19.3,15.2,14.2$. Elemental analysis: calcd (\%) for $\mathrm{C}_{23} \mathrm{H}_{20} \mathrm{~N}_{2} \mathrm{OS}_{2}$ (404.54): C 68.29, H 4.98; found: C 68.12, H 5.21. MS $(\mathrm{IE})=(\mathrm{M}) 404 \mathrm{~m} / \mathrm{z}$. 
2-Ethyl-4-methyl-5-(2-(2-methyl-5-(pyridin-3-yl)thiazol-4-yl)phenyl)thiazole (26): Following the general procedure $\mathbf{C}$ using 4-(2-Bromophenyl)-2-methyl-5-(pyridin-3-yl)thiazole (11) (166 mg, $0.5 \mathrm{mmol}$, 1 equiv.) and 2-ethyl-4-methylthiazole (127 mg, $1 \mathrm{mmol}, 2$ equiv.), the residue was purified by flash chromatography on silica gel (EtOAc, 100) to afford the desired compound $\mathbf{2 6}(155 \mathrm{mg}, 82 \%)$ as brown oil: ${ }^{1} \mathrm{H}$ NMR (400 MHz, DMSO- $\left.d_{6}\right) \delta(\mathrm{ppm}) \delta 8.40(\mathrm{~d}, J=3.8 \mathrm{~Hz}, 1 \mathrm{H}), 7.97$ (s, $\left.1 \mathrm{H}\right), 7.67(\mathrm{~d}, J=6.4 \mathrm{~Hz}$, 1H), $7.59-7.40(\mathrm{~m}, 2 \mathrm{H}), 7.30(\mathrm{~d}, J=7.2 \mathrm{~Hz}, 1 \mathrm{H}), 7.24(\mathrm{~d}, J=4.7 \mathrm{~Hz}, 1 \mathrm{H}), 7.20-7.14(\mathrm{~m}, 1 \mathrm{H}), 2.76(\mathrm{q}$, $J=7.4 \mathrm{~Hz}, 2 \mathrm{H}), 2.72(\mathrm{~s}, 3 \mathrm{H}), 1.64(\mathrm{~s}, 3 \mathrm{H}), 1.18(\mathrm{t}, J=7.5 \mathrm{~Hz}, 3 \mathrm{H}) .{ }^{13} \mathrm{C}$ NMR $\left(75 \mathrm{MHz}, \mathrm{DMSO}-d_{6}\right) \delta$ (ppm) 170.2, 165.2, 149.4, 148.8, 148.1, 147.8, 135.5, 134.9, 131.9, 131.7, 131.0, 130.7, 129.3, 129.0, $128.8,128.0,124.2,26.3,19.4,15.4,14.5$. Elemental analysis: calcd (\%) for $\mathrm{C}_{21} \mathrm{H}_{19} \mathrm{~N}_{3} \mathrm{~S}_{2}(377.52)$ : $\mathrm{C}$ 66.81, H 5.07; found: C 67.06, H 4.85. MS (IE) $=(\mathrm{M}+1) 378 \mathrm{~m} / \mathrm{z}$.

2-Ethyl-4-methyl-5-(2-(2-methyl-5-(quinolin-3-yl)thiazol-4-yl)phenyl)thiazole (27): Following the general procedure $\mathbf{C}$ using 4-(2-bromophenyl)-2-methyl-5-(quinolin-3-yl)thiazole (12) (191 mg, 0.5 mmol, 1 equiv.) and 2-ethyl-4-methylthiazole (127 mg, $1 \mathrm{mmol}, 2$ equiv.), the residue was purified by flash chromatography on silica gel (pentane-EtOAc, 40-60) to afford the desired compound 27 (182 mg, $85 \%)$ as brown oil: ${ }^{1} \mathrm{H}$ NMR $\left(400 \mathrm{MHz}, \mathrm{DMSO}-d_{6}\right) \delta(\mathrm{ppm}) \delta 8.19(\mathrm{~d}, J=1.9 \mathrm{~Hz}, 1 \mathrm{H}), 7.93(\mathrm{~d}, J=8.4$ $\mathrm{Hz}, 1 \mathrm{H}), 7.81(\mathrm{~d}, J=8.0 \mathrm{~Hz}, 1 \mathrm{H}), 7.78-7.72(\mathrm{~m}, 3 \mathrm{H}), 7.63-7.53(\mathrm{~m}, 2 \mathrm{H}), 7.48(\mathrm{t}, J=7.3 \mathrm{~Hz}, 1 \mathrm{H}), 7.23$ $(\mathrm{d}, J=7.5 \mathrm{~Hz}, 1 \mathrm{H}), 2.76(\mathrm{~s}, 3 \mathrm{H}), 2.59$ (q, $J=7.5 \mathrm{~Hz}, 2 \mathrm{H}), 1.41(\mathrm{~s}, 3 \mathrm{H}), 1.00(\mathrm{t}, J=7.5 \mathrm{~Hz}, 3 \mathrm{H}) .{ }^{13} \mathrm{C} \mathrm{NMR}$ $\left(75 \mathrm{MHz}, \mathrm{DMSO}-d_{6}\right) \delta(\mathrm{ppm}) 170.2,165.4,149.5,149.0,147.6,146.8,134.9,134.5,132.1,131.8,130.9$, $130.4,129.3,129.0,128.9,128.8,128.5,127.7,125.2,26.2,19.5,15.1,14.0$. Elemental analysis: calcd (\%) for $\mathrm{C}_{25} \mathrm{H}_{21} \mathrm{~N}_{3} \mathrm{~S}_{2}$ (427.58): C 70.23, H 4.95; found: C 70.29, H 5.12. MS (IE) $=(\mathrm{M}) 427 \mathrm{~m} / \mathrm{z}$.

3-(4-(2-(2-Isobutylthiazol-5-yl)phenyl)-2-methylthiazol-5-yl)benzonitrile (28): Following the general procedure C using 3-(4-(2-bromophenyl)-2-methylthiazol-5-yl)benzonitrile (7) (178 mg, $0.5 \mathrm{mmol}, 1$ equiv.) and 2-isobutylthiazole (141 mg, $1 \mathrm{mmol}, 2$ equiv.), the residue was purified by flash chromatography on silica gel (pentane-EtOAc, 70-30) to afford the desired compound 28 (139 $\mathrm{mg}, 67 \%$ ) as brown oil: ${ }^{1} \mathrm{H} \mathrm{NMR}\left(400 \mathrm{MHz}, \mathrm{CDCl}_{3}\right) \delta(\mathrm{ppm}) 7.51(\mathrm{dd}, J=2.4,5.1 \mathrm{~Hz}, 1 \mathrm{H}), 7.46-7.37(\mathrm{~m}, 4 \mathrm{H})$, 
$7.21(\mathrm{~d}, J=7.8 \mathrm{~Hz}, 1 \mathrm{H}), 7.17(\mathrm{~d}, J=5.4 \mathrm{~Hz}, 1 \mathrm{H}), 7.10(\mathrm{~d}, J=7.9 \mathrm{~Hz}, 1 \mathrm{H}), 7.02(\mathrm{~s}, 1 \mathrm{H}), 2.75(\mathrm{~s}, 3 \mathrm{H}), 2.73$ $(\mathrm{d}, J=7.1 \mathrm{~Hz}, 2 \mathrm{H}), 2.08-1.89(\mathrm{~m}, 1 \mathrm{H}), 0.94(\mathrm{~d}, J=6.6 \mathrm{~Hz}, 6 \mathrm{H}) .{ }^{13} \mathrm{C} \mathrm{NMR}\left(75 \mathrm{MHz}, \mathrm{CDCl}_{3}\right) \delta(\mathrm{ppm})$ $170.4,165.1,149.8,139.9,136.1,133.2,132.9,132.4,132.1,131.6,131.2,131.0,130.9,130.3,129.3$, $129.3,128.7,118.1,112.7,42.1,29.7,22.2,19.4$. Elemental analysis: calcd (\%) for $\mathrm{C}_{24} \mathrm{H}_{21} \mathrm{~N}_{3} \mathrm{~S}_{2}(415.57)$ : C 69.37, H 5.09; found: C 69.44, H 5.21. MS (IE) $=(\mathrm{M}) 415 \mathrm{~m} / \mathrm{z}$.

2-Methyl-4-(2-(5-pentylthiophen-2-yl)phenyl)-5-(quinolin-3-yl)thiazole (29): Following the general procedure C using 4-(2-bromophenyl)-2-methyl-5-(quinolin-3-yl)thiazole (12) (191 mg, 0.5 mmol, 1 equiv.) and 2- $n$-pentylthiophene (155 $\mathrm{mg}, 1 \mathrm{mmol}, 2$ equiv.), the residue was purified by flash chromatography on silica gel (pentane-EtOAc, 80-20) to afford the desired compound 29 (159 mg, 70\%) as yellow oil: ${ }^{1} \mathrm{H}$ NMR $\left(400 \mathrm{MHz}, \mathrm{CDCl}_{3}\right) \delta(\mathrm{ppm}){ }^{1} \mathrm{H}$ NMR $\left(400 \mathrm{MHz}, \mathrm{CDCl}_{3}\right) \delta{ }^{1} \mathrm{H} \mathrm{NMR}(400 \mathrm{MHz}$, $\left.\mathrm{CDCl}_{3}\right) \delta 8.47(\mathrm{~s}, 1 \mathrm{H}), 7.99(\mathrm{~d}, J=8.4 \mathrm{~Hz}, 1 \mathrm{H}), 7.64(\mathrm{dd}, J=1.5,6.8 \mathrm{~Hz}, 1 \mathrm{H}), 7.60(\mathrm{~d}, J=1.9 \mathrm{~Hz}, 1 \mathrm{H})$, $7.57-7.53(\mathrm{~m}, 2 \mathrm{H}), 7.46(\mathrm{t}, J=7.5 \mathrm{~Hz}, 1 \mathrm{H}), 7.39-7.32(\mathrm{~m}, 3 \mathrm{H}), 6.36(\mathrm{~d}, J=3.5 \mathrm{~Hz}, 1 \mathrm{H}), 6.24(\mathrm{~d}, J=$ $3.5 \mathrm{~Hz}, 1 \mathrm{H}), 2.81(\mathrm{~s}, 3 \mathrm{H}), 2.55(\mathrm{t}, J=7.6 \mathrm{~Hz}, 2 \mathrm{H}), 1.53-1.40(\mathrm{~m}, 2 \mathrm{H}), 1.35-1.20(\mathrm{~m}, 4 \mathrm{H}), 0.88(\mathrm{t}, J=$ $7.0 \mathrm{~Hz}, 3 \mathrm{H}) .{ }^{13} \mathrm{C} \mathrm{NMR}\left(100 \mathrm{MHz}, \mathrm{CDCl}_{3}\right) \delta(\mathrm{ppm}) 164.6,150.6,149.9,146.7,139.6,134.6,134.5,132.8$, $131.2,130.8,130.0,129.5,129.1,129.0,127.7,127.7,127.5,126.9,125.5,125.2,123.8,31.3,31.2,29.9$, 22.4, 19.5, 14.0. Elemental analysis: calcd (\%) for $\mathrm{C}_{28} \mathrm{H}_{26} \mathrm{~N}_{2} \mathrm{~S}_{2}$ (454.65): C 73.97, H 5.76; found: C 74.11, H 5.69. HRMS (ESI) m/z: [M + Na $]^{+}$Calcd for $\mathrm{C}_{28} \mathrm{H}_{26} \mathrm{~N}_{2} \mathrm{NaS}_{2}$ 477.14351; Found 477.1435.

4-(2-(Imidazo[1,2-a]pyridin-3-yl)phenyl)-2-methyl-5-(quinolin-3-yl)thiazole $\quad(30):$ Following the general procedure $\mathbf{C}$ using 4-(2-bromophenyl)-2-methyl-5-(quinolin-3-yl)thiazole (12) (191 mg, 0.5 mmol, 1 equiv.) and imidazo[1,2-a]pyridazine (118 mg, $1 \mathrm{mmol}, 2$ equiv.), the residue was purified by flash chromatography on silica gel $\left(\mathrm{CH}_{2} \mathrm{Cl}_{2}-\mathrm{MeOH}, 95-5\right)$ to afford the desired compound 30 (172 mg, 82\%) as brown oil: ${ }^{1} \mathrm{H}$ NMR (400 MHz, $\left.\mathrm{CDCl}_{3}\right) \delta(\mathrm{ppm}) 8.22(\mathrm{~d}, J=2.2 \mathrm{~Hz}, 1 \mathrm{H}), 7.89(\mathrm{~d}, J=7.0 \mathrm{~Hz}$, 1H), $7.76(\mathrm{~d}, J=8.4 \mathrm{~Hz}, 1 \mathrm{H}), 7.58-7.49(\mathrm{~m}, 3 \mathrm{H}), 7.47-7.40(\mathrm{~m}, 1 \mathrm{H}), 7.37-7.32(\mathrm{~m}, 2 \mathrm{H}), 7.31-7.24$ (m, 2H), $7.20(\mathrm{~d}, J=8.8 \mathrm{~Hz}, 1 \mathrm{H}), 7.12(\mathrm{~s}, 1 \mathrm{H}), 6.54(\mathrm{t}, J=7.0,7.7 \mathrm{~Hz}, 1 \mathrm{H}), 5.91(\mathrm{t}, J=6.1,7.4 \mathrm{~Hz}, 1 \mathrm{H})$, $2.74(\mathrm{~s}, 3 \mathrm{H}) .{ }^{13} \mathrm{C} \mathrm{NMR}\left(100 \mathrm{MHz}, \mathrm{CDCl}_{3}\right) \delta(\mathrm{ppm}) 165.3,149.5,149.0,146.8,144.7,134.9,134.1,133.0$, 
$132.0,130.3,129.7,129.5,129.1,129.1,128.8,127.8,126.8,126.8,124.4,123.5,123.3,121.8,116.7$,

111.3, 19.4. Elemental analysis: calcd (\%) for $\mathrm{C}_{26} \mathrm{H}_{18} \mathrm{~N}_{4} \mathrm{~S}$ (418.52): C 74.62, H 4.34; found: C 74.37, $\mathrm{H}$ 4.22. $\mathrm{MS}(\mathrm{IE})=(\mathrm{M}) 418 \mathrm{~m} / \mathrm{z}$.

\section{ASSOCIATED CONTENT}

The Supporting Information is available free of charge on the ACS Publications website. Characterization data, ${ }^{1} \mathrm{H}$ and ${ }^{13} \mathrm{C}$ NMR spectra for all new compounds.

\section{AUTHOR INFORMATION}

\section{Corresponding Author}

* jean-francois.soule@univ-rennes1.fr, henri.doucet@univ-rennes1.fr

\section{ACKNOWLEDGMENT}

We thank the CNRS, "Rennes Metropole" and French Scientific Ministry of Higher Education for providing financial support.

\section{REFERENCES}

1. (a) Anthony, J. E., Chem. Rev. 2006, 106, 5028-5048; (b) Wu, J.; Pisula, W.; Müllen, K., Chem. Rev. 2007, 107, 718-747; (c) He, Z.; Xu, X.; Zheng, X.; Ming, T.; Miao, Q., Chem. Sci. 2013, 4, 4525-4531.

2. (a) Davy, N. C.; Man, G.; Kerner, R. A.; Fusella, M. A.; Purdum, G. E.; Sezen, M.; Rand, B. P.; Kahn, A.; Loo, Y.-L., Chem. Mater. 2016, 28, 673-681; (b) Zhou, W.; Zhang, Z.-G.; Ma, L.; Li, Y.; Zhan, X., Sol. Energy Mater. Sol. Cells 2013, 112, 13-19.

3. Fujikawa, T.; Segawa, Y.; Itami, K., J. Am. Chem. Soc. 2016, 138, 3587-3595.

4. (a) Daigle, M.; Picard-Lafond, A.; Soligo, E.; Morin, J.-F., Angew. Chem. Int. Ed. Engl. 2016, 55, 2042-2047;

(b) Zhang, Q.; Peng, H.; Zhang, G.; Lu, Q.; Chang, J.; Dong, Y.; Shi, X.; Wei, J., J. Am. Chem. Soc. 2014, 136, 5057-5064.

5. (a) Gu, X.; Wang, H.; Roose, J.; He, Z.; Zhou, Y.; Yan, Y.; Cai, Y.; Shi, H.; Zhang, Y.; Sung, H. H. Y.; Lam, J. W. Y.; Miao, Q.; Zhao, Y.; Wong, K. S.; Williams, I. D.; Tang, B. Z., Chem. Eur. J. 2015, 21, 17973-17980; (b) Skonieczny, K.; Gryko, D. T., J. Org. Chem. 2015, 80, 5753-5763; (c) Grzybowski, M.; Deperasinska, I.; Chotkowski, M.; Banasiewicz, M.; Makarewicz, A.; Kozankiewicz, B.; Gryko, D. T., Chem. Commun. 2016, 52 , 5108-5111; (d) Krzeszewski, M.; Swider, P.; Dobrzycki, L.; Cyranski, M. K.; Danikiewicz, W.; Gryko, D. T., Chem. Commun. 2016, 52, 11539-11542. 
6. (a) Dondoni, A.; Marra, A., Chem.Rev. 2004, 104, 2557-2600; (b) Kashyap, S. J.; Garg, V. K.; Sharma, P. K.; Kumar, N.; Dudhe, R.; Gupta, J. K., Med. Chem.Res. 2012, 21, 2123-2132; (c) Ayati, A.; Emami, S.; Asadipour, A.; Shafiee, A.; Foroumadi, A., Eur. J. Med. Chem. 2015, 97, 699-718.

7. Cote, B.; Boulet, L.; Brideau, C.; Claveau, D.; Ethier, D.; Frenette, R.; Gagnon, M.; Giroux, A.; Guay, J.; Guiral, S.; Mancini, J.; Martins, E.; Masse, F.; Methot, N.; Riendeau, D.; Rubin, J.; Xu, D.; Yu, H.; Ducharme, Y.; Friesen, R. W., Bioorg. Med. Chem. Lett. 2007, 17, 6816-6820.

8. (a) Moreno, I.; Tellitu, I.; SanMartin, R.; Badia, D.; Carrillo, L.; Dominguez, E., Tetrahedron Lett. 1999, 40, 5067-5070; (b) Moreno, I.; Tellitu, I.; San Martin, R.; Dominguez, E., Svnlett 2001, 1161-1163; (c) Moreno, I.; Tellitu, I.; Dominguez, E.; SanMartin, R., Eur. J. Org. Chem. 2002, 2126-2135.

9. Al-Awadi, H.; Ibrahim, M. R.; Al-Awadi, N. A.; Ibrahim, Y. A., Tetrahedron 2007, 63, 12948-12953.

10. (a) Kakiuchi, F.; Kochi, T., Svnthesis 2008, 3013-3039; (b) White, M. C., Synlett 2012, 23, 2746-2748; (c) Satoh, T.; Miura, M., Synthesis 2010, 3395-3409; (d) Yuan, K.; Soulé, J.-F.; Doucet, H., ACS Catal. 2015, 5, 978-991.

11. (a) Ackermann, L.; Vicente, R.; Kapdi, A. R., Angew. Chem. Int. Ed. 2009, 48, 9792-9826; (b) Bellina, F.; Rossi, R., Tetrahedron 2009, 65, 10269-10310; (c) Rossi, R.; Bellina, F.; Lessi, M.; Manzini, C., Adv. Synth. Catal. 2014, 356, 17-117; (d) Bheeter, C. B.; Chen, L.; Soulé, J.-F.; Doucet, H., Catal. Sci. Technol. 2016, 6, 2005-2049.

12. (a) Beccalli, E. M.; Broggini, G.; Martinelli, M.; Sottocornola, S., Chem. Rev. 2007, 107, 5318-5365; (b) Cho, S. H.; Kim, J. Y.; Kwak, J.; Chang, S., Chem. Soc. Rev. 2011, 40, 5068-5083.

13. (a) Chen, X.; Engle, K. M.; Wang, D.-H.; Yu, J.-Q., Angew. Chem. Int.Ed. 2009, 48, 5094-5115; (b) Lyons, T. W.; Sanford, M. S., Chem. Rev. 2010, 110, 1147-1169; (c) Sun, C.-L.; Li, B.-J.; Shi, Z.-J., Chem. Commun. 2010, 46, 677-685.

14. (a) Arockiam, P. B.; Bruneau, C.; Dixneuf, P. H., $\underline{\text { Chem. Rev }}$ 2012, 112, 5879-5918; (b) Kozhushkov, S. I.; Ackermann, L., Chem. Sci. 2013, 4, 886-896; (c) Li, B.; Dixneuf, P. H., Chem. Soc. Rev. 2013, 42, 5744-5767.

15. (a) Colby, D. A.; Bergman, R. G.; Ellman, J. A., Chem. Rev. 2009, 110, 624-655; (b) Hirano, K.; Miura, M., Chem. Lett. 2015, 44, 878-873.

16. (a) Kuhl, N.; Hopkinson, M. N.; Wencel-Delord, J.; Glorius, F., Angew. Chem. Int. Ed. 2012, 51, 10236-10254;

(b) Zhang, M.; Zhang, Y.; Jie, X.; Zhao, H.; Li, G.; Su, W., Org. Chem. Front. 2014, 1, 843-895; (c) Yadav, M. R.; Rit, R. K.; Shankar, M.; Sahoo, A. K., Asian J. Org. Chem. 2015, 4, 846-864.

17. Li, B.-J.; Shi, Z.-J., Chem. Soc.Rev. 2012, 41, 5588-5598.

18. Yamaguchi, J.; Yamaguchi, A. D.; Itami, K., Angew. Chem. Int.Ed. 2012, 51, 8960-9009.

19. Iwasaki, M.; Iino, S.; Nishihara, Y., Org. Lett. 2013, 15, 5326-5329.

20. García-López, J.-A.; Greaney, M. F., Org. Lett. 2014, 16, 2338-2341.

21. (a) Saito, K.; Chikkade, P. K.; Kanai, M.; Kuninobu, Y., Chem. Eur. J. 2015, 21, 8365-8368; (b) Iitsuka, T.; Hirano, K.; Satoh, T.; Miura, M., J. Org.Chem. 2015, 80, 2804-2814; (c) Schubert, M.; Trosien, S.; Schulz, L.; Brandscheid, C.; Schollmeyer, D.; Waldvogel, S. R., Eur. J. Org. Chem. 2014, 2014, 7091-7094.

22. Schnapperelle, I.; Bach, T., Chem. Eur. J. 2014, 20, 9725-9732.

23. Hagui, W.; Besbes, N.; Srasra, E.; Roisnel, T.; Soulé, J.-F.; Doucet, H., Org. Lett. 2016, 18, 4182-4185.

24. (a) Zhang, M.; Zhang, S.; Liu, M.; Cheng, J., Chem.Commun. 2011, 47, 11522-11524; (b) Yu, X.; Li, X.; Wan, B., Org. Biomol. Chem. 2012, 10, 7479-7482; (c) Jafarpour, F.; Olia, M. B. A.; Hazrati, H., Adv. Synth. Catal. 2013, 
355, 3407-3412; (d) Miao, T.; Li, P.; Wang, G.-W.; Wang, L., Chem. Asian J. 2013, 8, 3185-3190; (e) Yuan, K.; Doucet, H., Chem. Sci. 2014, 5, 392-396; (f) Jin, R.; Yuan, K.; Chatelain, E.; Soule, J.-F.; Doucet, H., Adv. Synth. Catal. 2014, 356, 3831-3841; (g) Hfaiedh, A.; Yuan, K.; Ben Ammar, H.; Ben Hassine, B.; Soulé, J.-F.; Doucet, H., ChemSusChem 2015, 8, 1794-1804; (h) Skhiri, A.; Beladhria, A.; Yuan, K.; Soulé, J.-F.; Ben Salem, R.; Doucet, H., Eur. J. Org. Chem. 2015, 4428-4436.

25. (a) Hagui, W.; Yuan, K.; Besbes, N.; Srasra, E.; Soulé, J.-F.; Doucet, H., ChemCatChem 2015, 7, 3544-3554; (b) Xu, X.; Zhao, L.; Li, Y.; Soulé, J.-F.; Doucet, H., Adv. Svnth. Catal. 2015, 357, 2869-2882.

26. Fan, S.; Chen, Z.; Zhang, X., Org. Lett. 2012, 14, 4950-4953.

27. For intramolecular Pd-catalyzed direct arylation see: Campeau, L.-C.; Parisien, M.; Leblanc, M.; Fagnou, K., J. Am. Chem. Soc. 2004, 126, 9186-9187.

28. Distefano, G.; Granozzi, G.; Olivato, P. R., J. Chem. Soc. Perkin Trans. 2 1985, 2037-2040.

29. (a) Campeau, L.-C.; Rousseaux, S.; Fagnou, K., J.Am. Chem. Soc. 2005, 127, 18020-18021; (b) GarciaCuadrado, D.; Braga, A. A. C.; Maseras, F.; Echavarren, A. M., J.Am. Chem. Soc. 2006, 128, 1066-1067; (c) Hostyn, S.; Maes, B. U. W.; Van Baelen, G.; Gulevskaya, A.; Meyers, C.; Smits, K., Tetrahedron 2006, 62, 46764684; (d) Garcia-Cuadrado, D.; de Mendoza, P.; Braga, A. A. C.; Maseras, F.; Echavarren, A. M., J. Am. Chem. Soc. 2007, 129, 6880-6886; (e) Marquise, N.; Harford, P. J.; Chevallier, F.; Roisnel, T.; Dorcet, V.; Gagez, A.-L.; Sable, S.; Picot, L.; Thiery, V.; Wheatley, A. E. H.; Gros, P. C.; Mongin, F., Tetrahedron 2013, 69, 10123-10133.

30. Cantat, T.; Génin, E.; Giroud, C.; Meyer, G.; Jutand, A., J. Organomet. Chem. 2003, 687, 365-376. 